\title{
Segregación socioeconómica en las escuelas de Lima Metropolitana
}

Pedro Garret

Oficina de Medición de la Calidad de los Aprendizajes (UMC) - Ministerio de Educación

https://orcid.org/0000-0002-5213-1331

usoresultadosumc@minedu.gob.pe

\section{Liliana Miranda Molina}

Grupo de Análisis para el Desarrollo (Grade)

https://orcid.org/0000-0001-9716-7410

lmiranda@grade.org.pe

\section{Manuel Marcos Balabarca}

Universidad Complutense de Madrid (UCM)

https://orcid.org/0000-0001-6252-035X

manumarc@ucm.es

\section{Andrés Christiansen}

International Association for the Evaluation

of Educational Achievement (IEA)

https://orcid.org/0000-0003-2692-7843

andres.christiansen@iea-hamburg.de

Recibido: 02/11/2021

Aprobado: 07/12/2021 


\title{
Segregación socioeconómica en las escuelas de Lima Metropolitana
}

\begin{abstract}
Resumen
Este estudio busca profundizar en el conocimiento de la segregación escolar por nivel socioeconómico (NSE) en Lima Metropolitana. Para ello, se estimó la magnitud de la segregación socioeconómica en las escuelas de este ámbito considerando el nivel educativo (primaria/secundaria), tipo de gestión (estatal/no estatal), costo de pensiones (escuelas no estatales) y zonificación geográfica. Se utilizó el índice socioeconómico de los estudiantes recogido en la Evaluación Censal de Estudiantes 2018. La estimación de la segregación se realizó a través del Î́ndice de Disimilitud, del Coeficiente de Correlación Intraclase y del Îndice de Aislamiento. También, se categorizó a las escuelas por su condición de segregación socioeconómica.

Aunque los resultados varían según el índice utilizado, en general, se encontró que existe una mayor segregación socioeconómica en las escuelas primarias que en las secundarias. La segregación no es un fenómeno uniforme, pues se manifiesta de manera diferenciada según el tipo de gestión de la escuela. Si bien se registran fuertes indicios de segregación tanto en escuelas estatales como no estatales, en estas últimas, son mayores para ambos niveles educativos. Respecto a las zonas geográficas, los índices de segregación tienden a valores más elevados en aquellas que concentran una mayor proporción de estudiantes de NSE más alto. No obstante, los estudiantes con NSE más bajo tienen una mayor probabilidad de coincidir con pares de su mismo estatus socioeconómico que asisten a escuelas de los distritos periféricos de Lima Metropolitana. En todos los distritos analizados, al menos la tercera parte de las escuelas primarias y la cuarta parte de las de secundaria se encuentran en condición de segregación.

Palabras clave: Segregación escolar, nivel socioeconómico, educación privada, integración educativa, Lima
\end{abstract}




\title{
Socioeconomic Segregation in the Schools of the Lima Metropolitan Area
}

\begin{abstract}
This study aims to deepen the knowledge of school segregation by socioeconomic status (SES) in the Lima Metropolitan Area. To do this, the magnitude of socioeconomic segregation in schools of this area was estimated by educational level (primary/secondary), type of management (public/private), school fees (private schools), and zoning. The students' socioeconomic index collected in the $2018 \mathrm{Stu}$ dent Census Assessment was used. The estimation of segregation was carried out through the Dissimilarity Index, the Intraclass Correlation Coefficient, and the Isolation Index. Schools were also categorized by their socioeconomic segregation status.

Although the results vary according to the index used, it was found that there is a stronger socioeconomic segregation in primary schools than in secondary schools. Segregation is not a uniform phenomenon, as it varies according to the type of school management. While there are strong indications of segregation in both public and private schools, in the latter, they are higher for primary and secondary schools. Regarding geographical areas, segregation indices tend to be higher in those that concentrate a greater proportion of students with higher SES. However, students with a lower SES are more likely to coincide with peers of the same socioeconomic status who attend schools in the peripheral districts of the Lima Metropolitan Area. In all the districts analyzed, at least a third of the primary schools and a quarter of the secondary schools are segregated.
\end{abstract}

Keywords: School segregation, socioeconomic status, private education, school integration, Lima 


\section{Introducción}

En los últimos años, un conjunto creciente de estudios ha aportado evidencias sólidas acerca de la alta incidencia de la segregación escolar en el Perú (Benavides et al., 2014; Balarin y Escudero, 2019; Cueto et al., 2016; Murillo y Carrillo, 2020a, 2020b; OECD, 2019). Ello resulta preocupante por sus efectos negativos: debilita la experiencia formativa integral de los estudiantes, acentúa las desigualdades en los resultados académicos en los grupos más vulnerables y reduce la efectividad de las políticas de mejora educativa (Alegre, 2010; Bellei, 2013; Murillo y Carrillo, 2021; Valenzuela et al., 2010).

Las estimaciones y los análisis sobre segregación escolar por nivel socioeconómico realizados en la última década en el Perú permiten comprender buena parte de las dinámicas sociales y educativas que generan esta problemática. Un siguiente nivel en el análisis debería incorporar los ámbitos subnacionales y regionales.

Para contribuir a ese propósito, el presente artículo busca analizar el proceso de segregación escolar por nivel socioeconómico en Lima Metropolitana $(\mathrm{LM})^{1}$. Estudiar el fenómeno de la segregación en la capital es relevante por varias razones. Lima es la ciudad que concentra a la mayor población del Perú (31\%) (Instituto Nacional de Estadística e Informática, 2021). Además, representa la tercera parte de la matrícula nacional de la educación básica y a la cuarta parte de la fuerza laboral docente del país. Por otro lado, en LM se encuentra el $46 \%$ de la matrícula privada ${ }^{2}$, mientras que, a nivel nacional, esta cifra alcanza al 25\% (Minedu, 2021).

La matrícula privada en LM se incrementó de manera significativa en las dos últimas décadas (Balarin et al., 2018; Cuenca, 2013), fenómeno que ha sido definido como un proceso de privatización por defecto (Balarin, 2016). Este crecimiento ha generado una oferta educativa caracterizada por su alta heterogeneidad en términos de condiciones socioeconómicas y de rendimiento académico. En LM, se concentra el mayor número de escuelas privadas de muy alto y bajo costo, aunque se ha identificado que "la mayoría de estudiantes que asisten a una escuela privada en esta ciudad están matriculados en una de bajo costo" (Minedu, 2018b, p. 23). Algunas investigaciones indican que el peso que ha adquirido la educación privada en el Perú, sobre todo de bajo costo, así

1. Lima Metropolitana incluye a la conurbación del centro urbano de la provincia de Lima y de la provincia constitucional del Callao en tanto conforman una sola trama urbana. Su división obedece solo a criterios administrativos.

2. Cabe indicar que, por efecto de la situación de emergencia por la COVID-19, la matrícula escolar en Lima Metropolitana tuvo variaciones inusuales tanto en primaria como en secundaria. Aproximadamente, 24959 estudiantes de primaria (17,81\%) cambiaron de escuela para el periodo 2019-2020, de los cuales 10634 estudiantes (7,58\%) cambiaron de una escuela no estatal urbana a una estatal urbana. Una tendencia similar se encontró para el nivel de secundaria. 
como la generalización de la libre elección de escuelas, estaría contribuyendo a la incidencia de la segregación (Balarin y Escudero, 2019; Benavides et al., 2014; Murillo et al., 2020).

Por último, uno de los factores que configura los procesos de segregación escolar es la segmentación residencial (Bonal y Bellei, 2019). El caso de LM se caracteriza por tener espacios ocupados por sectores sociales que comparten características de manera próxima (Carrillo et al., 2019; Pereyra, 2006). La segregación espacial se manifiesta en la medida en que la población más vulnerable se asienta en las zonas periféricas o "conos" de la ciudad, a diferencia de aquella con mayores recursos, que se ubica principalmente en las zonas de mayor desarrollo urbano. Diversos estudios sugieren que la población de renta alta tiende a ser más homogénea que el grupo en condición de vulnerabilidad (Talavera, 2019).

Todo lo señalado configura a LM como un escenario donde la segregación se encuentra presente en la conformación espacial de la ciudad, y donde la organización escolar presenta altas tasas de matrícula privada, la cual se caracteriza por su heterogeneidad socioeconómica y se refleja en el mercado educativo con escuelas privadas de diverso costo (Balarin et al., 2018; Minedu, 2018b). Estas características hacen relevante una mirada específica de LM sobre el fenómeno de la segregación escolar por nivel socioeconómico, diferenciada del resto del país.

\section{Revisión de la literatura}

La segregación es un fenómeno multidimensional. En ese sentido, existen diversas aproximaciones conceptuales sobre segregación. Por ello, esta puede adquirir significados variados, los cuales dependen del campo disciplinario desde el que se realiza. No obstante, los estudios de James y Taeuber (1985), y de Gorard y Taylor (2002) proveen una definición con cierto consenso. De acuerdo con estos autores, la segregación alude a la disparidad en la distribución de los rasgos o características individuales de los grupos sociales (género, lengua, grupo étnico, nivel socioeconómico) entre distintas unidades organizativas (escuelas, barrios, áreas residenciales) relacionadas a una determinada área geográfica o territorial.

La aproximación empírica de la segregación no es sencilla de sintetizar dada las dimensiones involucradas en un fenómeno tan complejo. Massey y Denton (1988), en su estudio clásico sobre segregación residencial, plantearon cinco dimensiones: uniformidad o igualdad, exposición, concentración, centralización y agrupamiento. En el campo de la investigación educativa sobre segregación escolar, se utilizan las dos primeras, de donde derivan los índices para su medición (Echenique y Fryer, 2007; Elacqua, 2012; Gorard y Taylor, 2002; Massey y Denton, 1988; Murillo, 2016; Valenzuela et al., 2010).

La dimensión de uniformidad se refiere a la disparidad en la distribución de estudiantes sobre la base de algún atributo social específico entre escuelas. 
En tal sentido, un grupo de estudiantes con ciertas características se encontrará segregado si su distribución entre las instituciones educativas resulta desigual. Por otro lado, la dimensión de exposición alude a la probabilidad de que estudiantes con ciertas características sociales se encuentren con sus pares de diferentes grupos sociales. De esta manera, la segregación de un grupo de estudiantes será evidente si la probabilidad de interacción con miembros de otros grupos sociales es baja, es decir, si se encuentra en una situación de aislamiento relativo en relación con dichos grupos (Allen y Vignoles, 2007; Bellei, 2013; Dupriez, 2010; Massey y Denton, 1988; Murillo, 2016).

Existen distintos índices para estimar la segregación escolar. Si se aborda la segregación desde la dimensión de la uniformidad, es posible identificar cinco índices, que son los más utilizados en las investigaciones: el índice de Disimilitud (Duncan); el índice de Gorard; el índice de Raíz Cuadrada (Hutchens); el índice de Brecha por Centiles; y el índice de Inclusión Socioeconómica, que se basa en modelos multinivel. Desde la dimensión de exposición, el índice más usado es el de Aislamiento (Allen y Vignoles, 2007; Duncan y Duncan, 1955; Massey y Denton, 1988; Murillo, 2016; Murillo y Graña Oliver, 2020).

La segregación escolar se define como la distribución desigual de los estudiantes en unas escuelas $u$ otras que toma como referencia sus atributos o características. Si bien existen múltiples tipos de segregación escolar, cabe considerar a las siguientes principales: la segregación racial y/o étnica, sea que considere la población de minorías étnicas o la población inmigrante extranjera; la segregación socioeconómica; y la segregación académica (Murillo, 2016). Si bien en Estados Unidos la segregación racial ha dominado la mayor parte de la investigación educativa, esto no ha ocurrido en otras partes del mundo (Bellei, 2013; Bonal y Bellei, 2019; Murillo y Graña Oliver, 2020; Valenzuela et al., 2010). De hecho, los recientes estudios sobre segregación escolar en América Latina y en Perú han centrado su atención en la segregación por nivel socioeconómico. Esta decisión se explica por la alta incidencia que tienen las desigualdades socioeconómicas en la región. No obstante, es probable que también se deba al hecho de que aproximarse a la frontera que define a los diversos grupos étnicos - más allá de la variable lingüística - para el análisis de la segregación escolar no resulta una tarea sencilla (Murillo, 2016; Valenzuela et al., 2010).

La segregación escolar puede ser analizada desde diferentes dimensiones: magnitud; escala; causas; dinámica espacial; consecuencias y efectos en el desempeño escolar, y en la cohesión e integración social; entre otros. La literatura sobre el tema identifica ciertos factores externos e internos que configuran los procesos de segregación educativa. Entre los primeros, se encuentran los aspectos contextuales, como las tendencias demográficas y territoriales (la segmentación residencial y los llamados efectos de vecindario), así como los aspectos culturales, que están relacionados con las motivaciones y valoraciones

94 I de las familias para elegir la escuela para sus hijos. Por su parte, los factores 
institucionales del propio sistema educativo se vinculan, entre otros aspectos, con los criterios formales o informales de los procesos de admisión, la composición del sector público y privado, las políticas compensatorias, y el rol del mercado en educación (Bellei, 2013; Bonal y Bellei, 2019).

En América Latina, se han llevado a cabo estudios comparados sobre segregación escolar por nivel socioeconómico basados en información proveniente de las evaluaciones estandarizadas internacionales (Arcidiácono et al., 2014; Benavides et al., 2014; Krüger, 2019; Murillo, 2016; Murillo y Martínez-Garrido, 2017; Murillo et al., 2018; Vazquez, 2016). Estos estudios han permitido cuantificar la magnitud de la segregación escolar de la región, estimar su evolución a lo largo de los últimos quince años, así como evidenciar el alto grado de incidencia que presenta dicho fenómeno en comparación con otras regiones del mundo. Los resultados de estas investigaciones constituyen una base importante para contrastar y profundizar los estudios a nivel de país.

En el caso peruano, diversas investigaciones han mostrado de manera consistente que un mayor nivel socioeconómico (NSE) de las familias tiene un efecto positivo y significativo en el desempeño escolar de los estudiantes (Benavides et al., 2014; Guadalupe et al., 2013; León y Collahua, 2016; Minedu, 2016; Miranda, 2008; OECD, 2019). Más aún, se ha observado que el efecto de las variables socioeconómicas en el desempeño escolar en el nivel escuela es mayor que el efecto en el nivel individual del estudiante. Es decir, la composición socioeconómica de los estudiantes explica en gran medida las diferencias en los resultados académicos. En esa línea, en los últimos años, se han desarrollado estudios específicos que muestran que el sistema educativo peruano se caracteriza por sus altos niveles de segregación escolar por NSE. A continuación, se describen brevemente estas investigaciones presentadas por nivel educativo.

En educación primaria, los estudios de Murillo (2016), Murillo y Martínez-Garrido (2017), y Murillo et al. (2020) utilizaron la información del Tercer Estudio Regional Comparativo y Explicativo de la Unesco desde una aproximación comparada, y lograron evidenciar que Perú es uno de los países con mayores niveles de segregación escolar por nivel socioeconómico en la región latinoamericana. Así, el análisis realizado por Murillo y Martínez-Garrido (2017), con base en el índice de Disimilitud, sitúa a Perú en el grupo de tres países con mayor segregación escolar por NSE de la región, de allí que el 35\% de las escuelas peruanas concentre a más del $50 \%$ de los estudiantes con mayores desventajas. Por otro lado, los resultados de Murillo et al. (2020) mostraron que el Perú está ubicado en el grupo de países que presentan un sistema público-privado altamente segregado, y que el creciente peso del sector privado parece estar contribuyendo con una mayor incidencia de la segregación en el sistema escolar. 
En el marco del Estudio Young Lives ${ }^{3}$, Cueto et al. (2016) buscaron estimar el efecto de la segregación -composición en el aula derivada de la proporción de padres con educación secundaria completa o más- sobre el rendimiento académico y los aspectos socioemocionales en sexto grado de primaria. El análisis arrojó que, para que las aulas mantuvieran la misma distribución que la muestra completa, se tendría que trasladar al 53\% de los estudiantes que tienen padres con mayor nivel educativo a un aula en otra escuela primaria para tener una distribución similar. Además, los resultados sugieren que los mayores niveles de segregación se encuentran en los extremos de la distribución.

Especialmente interesante es la investigación de Balarin y Escudero (2019), quienes, con la información proveniente de las evaluaciones nacionales de sexto grado de primaria, analizaron el efecto de los procesos de privatización sobre la segregación. En primer lugar, las autoras encontraron que la segregación urbana por NSE se incrementó en el periodo 2001 y 2013. En segundo lugar, identificaron una tendencia hacia la homogeneización, aunque no uniforme, durante el periodo estudiado en la composición social de las escuelas públicas y privadas. Los análisis evidenciaron que, mientras en el grupo con NSE más alto se registra una tendencia hacia la homogeneización en ambos tipos de escuelas, en el grupo de NSE medio, dicha tendencia es más fuerte en las escuelas privadas. Este fenómeno se explicaría por el probable traslado de los estudiantes con mayor NSE de escuelas estatales a las escuelas privadas. A diferencia de estos dos casos, en el caso del grupo con NSE más bajo, el proceso de homogeneización es más ligero. No obstante, notaron que las escuelas de este grupo en el trascurso del tiempo estudiado se volvieron más homogéneamente pobres en su composición de NSE. El estudio concluyó que, en el periodo estudiado, la acentuación de la segregación escolar socioeconómica en el ámbito peruano urbano, antes que la segregación espacial, pudo haber sido el resultado "de la manera descontrolada y desgobernada en que se han establecido las dinámicas educativas de elección escolar" tanto en el sector privado como público (Balarin y Escudero, 2019, p. 41).

Más recientemente, Murillo y Carrillo (2020a), tomando como base la Evaluación Censal de Estudiantes (ECE) de 2016, realizaron estimaciones sobre la magnitud de la segregación por nivel socioeconómico en el nivel primario para el conjunto del Perú y para cada una de sus regiones. En sus análisis, encontraron una alta magnitud de segregación escolar en Perú. El índice de Gorard tomó los valores de 0,50 en el conjunto del país; 0,46 en el ámbito urbano; 0,50 en Lima Metropolitana (LM); y 0,46 en Callao. De acuerdo con el índice de Aislamiento, los valores fueron de 0,49; 0,43; 0,39; y 0,44, respectivamente. Así, encontraron que LM es parte del grupo de regiones con los

3. Young Lives, conocido en Perú como Niños del Milenio, es un estudio longitudinal que busca comprender las causas y consecuencias de la pobreza infantil. Desde 2002, sigue a un grupo de 12,000 niños de cuatro países en desarrollo: Etiopía, India, Perú y Vietnam. 
mayores niveles de segregación registrados. Además, los autores identificaron que, en ambos índices, la segregación nacional es más alta en el grupo de estudiantes cuyas familias tienen un mayor NSE en comparación con aquellos que pertenecen a familias de menor NSE. Esta tendencia se cumple también para el caso de LM; de hecho, registra una hipersegregación para el 10\% de estudiantes con mayor NSE.

Los estudios comparados en educación secundaria, que tomaron como base la información de la evaluación internacional PISA, han presentado la misma tendencia evidenciada en la primaria. Por ejemplo, el análisis que realizan Benavides et al. (2014) concluyó que el sistema educativo peruano no solo es el que exhibe el valor de segregación más alto entre los países que participan en PISA, sino también que es el país que registra un mayor incremento en el periodo 2001 y 2009, en comparación con sus pares regionales de Argentina, Brasil, Chile y México. Estos hallazgos coinciden con los estudios de Krüger (2019), Murillo et al. (2018) y Vazquez (2016), que analizaron la segregación socioeconómica en el periodo 2000 y 2015 para los países participantes de PISA. En cuanto a Perú, estos estudios evidenciaron una clara tendencia al incremento de los niveles de segregación en el grupo de estudiantes con familias de menor nivel socioeconómico.

Las tendencias mostradas se confirman con los resultados de PISA 2018. En los análisis de segregación socioeconómica realizados por la OECD (2019), se señaló que, en Perú, tanto el índice de Aislamiento de los estudiantes favorecidos como el de los estudiantes desfavorecidos fueron mucho más altos que el promedio de la OECD. Asimismo, se sostuvo que esta situación podría explicarse por el alto grado de segregación residencial y por el creciente predominio de la educación privada en el país. Respecto a esta última afirmación, el estudio de Benavides et al. (2004) encontró que la segregación aumentó tanto en las escuelas públicas como en las privadas en el periodo 2001 y 2009. Los autores sugirieron que el incremento de la segregación en las escuelas públicas se debe entender en el marco de la ampliación de la oferta privada en los grupos menos desfavorecidos en el país.

Por su parte, Murillo y Carrillo (2020b) también han generado estimaciones sobre la magnitud de la segregación por NSE para la educación secundaria sobre la base de la ECE 2016, tanto para el conjunto del país como para cada una de sus regiones. En sus análisis, encontraron que la magnitud de la segregación escolar en Perú es alta. El índice de Gorard tomó los valores de 0,54 en el conjunto del país; 0,51 en el ámbito urbano; 0,44 en Lima; y 0,45 en Callao. De acuerdo con el índice de Aislamiento, los valores fueron de 0,50; 0,36; 0,39; y 0,37 , respectivamente. Además, los resultados del estudio mostraron que la segregación a nivel nacional es más alta en el grupo de estudiantes cuyas familias presentan un menor NSE. Sin embargo, en el caso de Lima, la tendencia es contraria, pues la mayor segregación se encuentra en los grupos de mayor NSE.

Los estudios presentados aportan sólidas evidencias que sostienen que el Perú registra altos niveles de segregación escolar por NSE, y que este fenómeno 
se ha incrementado en el tiempo. Respecto a esto último, las investigaciones indican que el peso que ha adquirido la educación privada, sobre todo de bajo costo, así como la generalización de la libre elección de escuelas, estarían contribuyendo con la incidencia de la segregación (Balarin, 2016; Balarin y Escudero, 2019; Benavides et al., 2014). Estos hallazgos demandan estudios en espacios y contextos más específicos, que consideren la heterogeneidad que caracteriza al país. Así, se podrán comprender mejor las configuraciones y dinámicas de la segregación socioeconómica. El presente artículo busca avanzar en esa dirección centrando su mirada en el ámbito de Lima Metropolitana.

Así, el objetivo de la investigación es estimar la magnitud de la segregación socioeconómica en las escuelas de LM considerando el nivel educativo (primaria/ secundaria), tipo de gestión (estatal/no estatal), costo de pensiones (escuelas no estatales) y zonificación geográfica con base en la conformación distrital. Un aspecto destacable del presente estudio es que, para el cálculo de los índices de segregación, en lugar de tomar como criterio determinados percentiles o cuartiles para identificar a los grupos "minoritarios", se optó por priorizar la distribución socioeconómica de los estudiantes y las escuelas. Con ello, se ha buscado reflejar de manera más precisa cómo se manifiesta la segregación escolar en los grupos más vulnerables y más privilegiados según su estatus socioeconómico.

\section{Método}

\section{Participantes}

La población objetivo corresponde a las escuelas estatales y no estatales del ámbito urbano $^{4}$ de Lima Metropolitana y de la provincia constitucional del Callao, las cuales participaron en la Evaluación Censal de Estudiantes (ECE) aplicada por el Ministerio de Educación de Perú (Minedu) en el año 2018. Dicha evaluación se llevó a cabo considerando a los estudiantes pertenecientes a $4{ }^{\circ}$ grado de primaria y a $2 .^{\circ}$ grado de secundaria (niveles $\operatorname{CINE}^{5} 1$ y CINE 2 , respectivamente). La población total se analizó de manera separada por grado evaluado.

Para el caso del grado correspondiente al nivel primaria, se contó con un total de 4399 escuelas, 1055 en el estrato estatal y 3344 en el no estatal, que comprenden un total de 126723 estudiantes con información sobre su condición socioeconómica: 64290 en el estrato estatal y 62433 en el no estatal. De modo similar, para el grado del nivel secundaria, se consideró un total de 2941 escuelas, de las cuales 758 pertenecen al estrato estatal y 2183 al no estatal, comprendiendo un total de 151258 estudiantes con información sobre su condición socioeconómica: 83960 en el estrato estatal y 67,298 en el no estatal.

4. El porcentaje de escuelas rurales en estos ámbitos es mínimo, entre 0,1\% y 0,2\%.

5. Clasificación internacional normalizada de la educación 


\section{Medición}

Para la construcción de los diferentes índices de segregación considerados en este estudio, se utilizó el índice socioeconómico (ISE) de los estudiantes. El ISE fue elaborado por la Oficina de Medición de la Calidad de los Aprendizajes (UMC) del Minedu. La información fue recogida durante la ECE 2018 a través de cuestionarios de factores asociados dirigidos a padres de familia en primaria y a estudiantes en secundaria (Minedu, 2018a).

La UMC utilizó el ISE para determinar cuatro niveles socioeconómicos (NSE): 1) muy bajo, 2) bajo, 3) medio y 4) alto. Para establecer los cortes entre estos NSE, se consideró la distribución socioeconómica de la Encuesta Nacional de Hogares (Enaho) y la propuesta por la Asociación Peruana de Empresas de Inteligencia de Mercado (Apeim). Así, el NSE muy bajo representa el 35\% inferior de la muestra nacional; el NSE bajo, el siguiente 25\%; el NSE medio, el siguiente 25\%; y el NSE alto, el $15 \%$ restante.

En el presente estudio, se considera esta distribución socioeconómica nacional, puesto que representa adecuadamente la composición socioeconómica de Lima Metropolitana. Además, se decidió agrupar en los análisis a los estudiantes que fueron clasificados en los NSE bajo y muy bajo. Esta agrupación se realizó porque ambos representan condiciones similares de vulnerabilidad y marginalidad respecto al acceso a servicios básicos y a la satisfacción de necesidades; se diferencian solo en el grado en que estas condiciones de pobreza son experimentadas.

Asimismo, se han considerado distintos estratos para el análisis de los resultados. El primero de ellos fue la gestión de la escuela. En segundo lugar, se tuvo en cuenta la tipología de escuelas privadas ${ }^{6}$ elaborada por el Minedu (2018b). Por último, se consideró la zonificación geográfica compuesta por diferentes distritos, para lo cual se tomaron como referencia las agrupaciones elaboradas por el Instituto Nacional de Estadística e Informática (2014) e Ipsos (2018). Estos estratos se detallan en la tabla 1.

6. Si bien esta tipología refiere a las escuelas no estatales, en el presente artículo, se ha decidido conservar la denominación dada en la fuente original. 
Tabla 1

Estratos considerados para los análisis

\begin{tabular}{|c|c|}
\hline Estratos & Descripción \\
\hline Gestión de la escuela & $\begin{array}{l}\text { Comprende el tipo de gestión administrativa de la } \\
\text { escuela. } \\
\text { Esta puede ser estatal o no estatal. }\end{array}$ \\
\hline Tipología de escuelas privadas & $\begin{array}{l}\text { Comprende una clasificación de escuelas privadas } \\
\text { según la relación entre las pensiones cobradas y el } \\
\text { ingreso promedio por trabajo de la región. Esta clasi- } \\
\text { ficación comprende cuatro tipos de escuela privada: } \\
\text { de bajo costo, de medio costo, de alto costo y de muy } \\
\text { alto costo. }\end{array}$ \\
\hline Zona geográfica & $\begin{array}{l}\text { Comprende la división básica del ordenamiento } \\
\text { territorial. Se consideran } 50 \text { distritos en Lima Metro- } \\
\text { politana y Callao, agrupados en siete zonas geográfi- } \\
\text { cas: Lima norte, Lima centro, Lima "moderna", Lima } \\
\text { este, Lima sur, Balnearios y Callao. }\end{array}$ \\
\hline
\end{tabular}

Para mayor información sobre la distribución socioeconómica en las escuelas de Lima Metropolitana, se sugiere revisar el anexo A.

\section{Procedimiento}

Sobre la base del ISE construido por el Ministerio de Educación (2018a), se elaboraron tres índices de segregación diferentes, aunque complementarios, y una categorización de escuelas. Así, se calcularon los siguientes indicadores: 1) índice de disimilitud, 2) coeficiente de correlación intraclase, 3 ) índice de aislamiento, y 4) categorías de segregación en la escuela.

\section{- Indice de disimilitud}

Este índice se utiliza para calcular el nivel de segregación dentro de cada una de las escuelas. Así, se toma como referencia la distribución que presenta un grupo socioeconómico determinado de estudiantes en su escuela respecto al resto de la población y del sistema educativo (Allen y Vignoles, 2007; Duncan y Duncan, 1955; Krüger, 2019). Matemáticamente, se expresa de la siguiente manera:

$$
D=\frac{1}{2} \sum_{i=1}^{N}\left|\frac{x_{1 i}}{X_{1}}-\frac{x_{2 i}}{X_{2}}\right|
$$


Donde el número de escuelas va en el rango de $i=1,2,3, \ldots, N, x_{1 i}$ representa el número de estudiantes pertenecientes al grupo de referencia dentro de la escuela $i, x_{2 i}$ es el resto de la población de estudiantes dentro de la escuela $i$. De modo similar, $X_{1}$ y $X_{2}$ representan el total de estudiantes del grupo de referencia y del resto de la población estudiantil que existen dentro del sistema educativo, respectivamente.

\section{- Coeficiente de correlación intraclase (CCI)}

Este indicador se calcula a partir de formular un modelo nulo multinivel considerando la estructura anidada de estudiantes (nivel 1) dentro de escuelas (nivel 2) con el índice socioeconómico de los estudiantes como variable criterio (Murillo, 2016; Murillo y Martínez-Garrido, 2016). Su cálculo se traduce en la varianza inter-escuelas dividida por la varianza total del índice socioeconómico. Matemáticamente, se expresa mediante la especificación de un modelo multinivel:

$$
Y_{i}=\beta_{0}+\mu_{j}+e_{i j}
$$

Donde $Y i$ representa el índice socioeconómico del estudiante $i, \mu_{j}$ es el indicativo del clúster escuela (nivel 2) y $e_{i j}$ el indicativo del estudiante (nivel 1). Así, el CCI se especifica de la siguiente manera:

$$
\rho=\frac{\sigma_{u}^{2}}{\sigma_{u}^{2}+\sigma_{e}^{2}}
$$

Donde $\sigma_{u}^{2}$ representa la varianza del error del nivel 2 y $\sigma_{e}^{2}$ la varianza del error del nivel 1. De acuerdo con lo anterior, se señala que, a mayores valores del CCI, mayor será el nivel de segregación.

\section{- Índice de aislamiento}

Este índice se enfoca en calcular la probabilidad que tienen los estudiantes de un mismo nivel socioeconómico de encontrarse en una misma escuela (Krüger, 2019; Murillo, 2016). Matemáticamente, se expresa de la siguiente manera:

$$
S=\sum_{i=1}^{N}\left[\frac{x_{1 i}}{X_{1}} \times \frac{x_{1 i}}{y_{i}}\right]
$$

Donde $x_{1 i}$ y $y_{1 i}$ representan al total de estudiantes que pertenecen a un NSE específico dentro de la escuela $i$ y el total de estudiantes en la escuela 
$i$, respectivamente, mientras que $X_{1}$ es el número total de estudiantes que pertenecen al NSE de referencia dentro del sistema educativo.

- Categorías de segregación en la escuela

Esta categorización se enfoca en identificar si una escuela se encuentra en condición de segregación socioeconómica. Partiendo de la distribución socioeconómica de sus estudiantes, se considera que una escuela se encuentra en condición de segregación cuando al menos la mitad de sus estudiantes pertenece a un nivel socioeconómico específico.

Se establecieron tres categorías relacionadas con la magnitud de la segregación en las escuelas: 1) cuando menos del 50\% de los estudiantes de una escuela posean el mismo NSE, esta no se encontrará en condición de segregación, pues atiende a una población heterogénea; 2) cuando una escuela tenga entre el 50\% y $90 \%$ de sus estudiantes en un mismo NSE, esta se encontrará en condición de segregación con una población homogénea; y 3) cuando más del $90 \%$ de los estudiantes de una escuela pertenezcan al mismo NSE, esta se encontrará en condición de segregación con una población muy homogénea. Estos puntos de corte fueron establecidos teniendo como referencia las recomendaciones de Mikulyuk y Braddock (2016), y Wilkes y Iceland (2004).

La mirada integrada de todos los indicadores estimados permitirá tener una mejor aproximación a la magnitud de la segregación socioeconómica en las escuelas de Lima Metropolitana. Por un lado, el índice de disimilitud y el coeficiente de correlación intraclase abordan la dimensión de uniformidad de la segregación, y dan cuenta del grado de desbalance en la distribución de los estudiantes entre escuelas en función de sus características socioeconómicas. Por otro lado, el índice de aislamiento refleja la dimensión de exposición de la segregación, es decir, la probabilidad de que estudiantes de distintos niveles socioeconómicos coincidan o no en una misma escuela. Estos índices otorgan pistas acerca de la magnitud de la segregación en el sistema educativo, pero no sobre en qué proporción se está presentando este fenómeno en las escuelas que lo componen. Por esa razón, esta investigación también incluye una categorización de escuelas según su grado de segregación socioeconómica, lo que permite enriquecer los análisis y, en consecuencia, la comprensión y descripción de esta problemática en Lima Metropolitana. 


\section{Resultados}

Los principales indicadores para analizar la dinámica de segregación urbana en Lima Metropolitana, desagregada por tipo de gestión de la escuela, tipología de escuelas privadas y zona geográfica, se presentan para los niveles de primaria (ver tabla 2) y secundaria (ver tabla 3). La misma información para todos los distritos de Lima Metropolitana se detalla en el anexo B.

$\mathrm{Al}$ analizar los resultados según gestión de la escuela, los indicadores reflejan que los niveles de segregación son mayores en las escuelas no estatales. Por ejemplo, los valores del índice de disimilitud son más altos para dicho tipo de escuelas tanto en primaria como en secundaria. En primaria, más de la mitad de los estudiantes de los NSE más bajos tendrían que cambiarse a escuelas de mayor estatus socioeconómico para alcanzar una distribución homogénea entre todas las escuelas no estatales de Lima Metropolitana, mientras que, en secundaria, deberían hacerlo poco más del $40 \%$ de los estudiantes de esos NSE.

No obstante, se encuentran diferencias al desagregar los valores del índice de disimilitud según tipo de escuela privada, especialmente en el caso de primaria. Para alcanzar una distribución socioeconómica proporcional en este nivel educativo, el número de estudiantes de NSE más bajos que tendrían que trasladarse a escuelas de mayor estatus socioeconómico es aproximadamente 4 de cada 10 de escuelas de bajo costo, 5 de cada 10 de las de medio costo, y 9 de cada 10 de las de alto y muy alto costo. En secundaria, dicha proporción de estudiantes es poco más de la tercera parte en las escuelas de bajo y medio costo, y alrededor de la mitad en las escuelas de alto y muy alto costo. Cuando se considera a los estudiantes de NSE alto, se aprecia una disminución en los valores del índice de disimilitud en ambos niveles educativos. En primaria, entre 39\% y $47 \%$ de estos estudiantes deberían cambiarse de institución educativa para lograr una distribución que los represente de manera proporcional en los distintos tipos de escuelas privadas, mientras que, en secundaria, dicha cantidad oscila entre $25 \%$ y $31 \%$. Estos resultados, al menos desde la dimensión de similitud de la segregación, evidenciarían un grado de diferenciación socioeconómica entre instituciones educativas no estatales más marcada en primaria, puesto que, en este nivel educativo, hay una relación más fuerte entre el estatus socioeconómico de los estudiantes y el costo de las escuelas a las que asisten. 
Tabla 2

Indicadores de segregación en $4 .^{\circ}$ grado de primaria

\begin{tabular}{|c|c|c|c|c|c|c|c|c|}
\hline \multirow[t]{2}{*}{ Estrato } & \multicolumn{2}{|c|}{ Disimilitud } & \multirow[t]{2}{*}{ CCI } & \multicolumn{2}{|c|}{ Aislamiento } & \multicolumn{3}{|c|}{ Grado de segregación } \\
\hline & $\begin{array}{c}\text { Muy } \\
\text { bajo y } \\
\text { Bajo }\end{array}$ & Alto & & $\begin{array}{c}\text { Muy } \\
\text { bajo y } \\
\text { Bajo }\end{array}$ & Alto & $\begin{array}{l}\text { Muy } \\
\text { homo- } \\
\text { géneo }\end{array}$ & $\begin{array}{l}\text { Ho- } \\
\text { mogé- } \\
\text { neo }\end{array}$ & $\begin{array}{l}\text { Hete- } \\
\text { rogé- } \\
\text { neo }\end{array}$ \\
\hline Estatal & 0,348 & 0,417 & 0,264 & 0,592 & 0,266 & $0,5 \%$ & $33,7 \%$ & $65,8 \%$ \\
\hline No estatal & 0,538 & 0,537 & 0,422 & 0,399 & 0,669 & $6,9 \%$ & $64,7 \%$ & $28,3 \%$ \\
\hline $\begin{array}{l}\text { Muy alto } \\
\text { costo }\end{array}$ & 0,933 & 0,470 & 0,051 & 0,015 & 0,981 & $96,6 \%$ & $3,4 \%$ & $0,0 \%$ \\
\hline Alto costo & 0,907 & 0,416 & 0,081 & 0,028 & 0,968 & $86,7 \%$ & $13,3 \%$ & $0,0 \%$ \\
\hline Medio costo & 0,511 & 0,385 & 0,219 & 0,159 & 0,730 & $11,3 \%$ & $77,6 \%$ & $11,0 \%$ \\
\hline Bajo costo & 0,397 & 0,387 & 0,230 & 0,429 & 0,380 & $0,0 \%$ & $61,9 \%$ & $38,1 \%$ \\
\hline Lima norte & 0,456 & 0,503 & 0,317 & 0,515 & 0,467 & $1,0 \%$ & $59,4 \%$ & $39,5 \%$ \\
\hline Lima centro & 0,478 & 0,511 & 0,359 & 0,413 & 0,585 & $3,7 \%$ & $59,0 \%$ & $37,3 \%$ \\
\hline $\begin{array}{l}\text { Lima } \\
\text { "moderna" }\end{array}$ & 0,692 & 0,675 & 0,476 & 0,329 & 0,846 & $36,8 \%$ & $48,2 \%$ & $14,9 \%$ \\
\hline Lima este & 0,433 & 0,525 & 0,331 & 0,563 & 0,430 & $0,8 \%$ & $51,6 \%$ & $47,6 \%$ \\
\hline Lima sur & 0,441 & 0,533 & 0,362 & 0,553 & 0,480 & $1,4 \%$ & $54,3 \%$ & $44,2 \%$ \\
\hline Balnearios & 0,432 & 0,520 & 0,338 & 0,754 & 0,208 & $4,2 \%$ & $54,2 \%$ & $41,7 \%$ \\
\hline Callao & 0,522 & 0,572 & 0,426 & 0,603 & 0,509 & $1,2 \%$ & $59,5 \%$ & $39,3 \%$ \\
\hline
\end{tabular}

En el caso de las escuelas estatales, para obtener tal distribución poblacional uniforme en términos socioeconómicos, aproximadamente $35 \%$ de los estudiantes de primaria de los NSE más bajos tendrían que pertenecer a escuelas de mayor estatus socioeconómico, mientras que al menos 4 de cada 10 estudiantes de NSE alto deberían ubicarse en escuelas de un estatus socioeconómico diferente. En secundaria, esta proporción es de 3 de cada 10 estudiantes, tanto para estudiantes de los NSE más bajos como para aquellos de NSE alto. Por lo tanto, al igual que en el caso de las escuelas no estatales, aquí también se evidencian niveles más altos de segregación socioeconómica en primaria en comparación con secundaria. 
Tabla 3

Indicadores de segregación en $2^{\circ}$ grado de secundaria

\begin{tabular}{|c|c|c|c|c|c|c|c|c|}
\hline \multirow[t]{2}{*}{ Estrato } & \multicolumn{2}{|c|}{ Disimilitud } & \multirow[t]{2}{*}{ CCI } & \multicolumn{2}{|c|}{ Aislamiento } & \multicolumn{3}{|c|}{ Grado de segregación } \\
\hline & $\begin{array}{c}\text { Muy } \\
\text { bajo y } \\
\text { Bajo }\end{array}$ & Alto & & $\begin{array}{c}\text { Muy } \\
\text { bajo y } \\
\text { Bajo }\end{array}$ & Alto & $\begin{array}{l}\text { Muy } \\
\text { homo- } \\
\text { géneo }\end{array}$ & $\begin{array}{l}\text { Ho- } \\
\text { mogé- } \\
\text { neo }\end{array}$ & $\begin{array}{l}\text { Hete- } \\
\text { rogé- } \\
\text { neo }\end{array}$ \\
\hline Estatal & 0,319 & 0,329 & 0,227 & 0,543 & 0,242 & $0,0 \%$ & $23,5 \%$ & $76,5 \%$ \\
\hline No estatal & 0,457 & 0,425 & 0,305 & 0,316 & 0,590 & $2,4 \%$ & $65,0 \%$ & $32,6 \%$ \\
\hline $\begin{array}{l}\text { Muy alto } \\
\text { costo }\end{array}$ & 0,524 & 0,283 & 0,082 & 0,039 & 0,922 & $58,6 \%$ & $41,4 \%$ & $0,0 \%$ \\
\hline Alto costo & 0,501 & 0,248 & 0,075 & 0,046 & 0,865 & $23,9 \%$ & $76,1 \%$ & $0,0 \%$ \\
\hline Medio costo & 0,379 & 0,306 & 0,157 & 0,173 & 0,602 & $1,1 \%$ & $76,2 \%$ & $22,7 \%$ \\
\hline Bajo costo & 0,339 & 0,312 & 0,164 & 0,376 & 0,337 & $0,2 \%$ & $56,2 \%$ & $43,6 \%$ \\
\hline Lima norte & 0,418 & 0,407 & 0,255 & 0,474 & 0,406 & $0,1 \%$ & $54,9 \%$ & $45,0 \%$ \\
\hline Lima centro & 0,390 & 0,421 & 0,236 & 0,371 & 0,477 & $0,5 \%$ & $56,4 \%$ & $43,1 \%$ \\
\hline $\begin{array}{l}\text { Lima } \\
\text { "moderna" }\end{array}$ & 0,548 & 0,512 & 0,275 & 0,284 & 0,720 & $11,2 \%$ & $71,4 \%$ & $17,4 \%$ \\
\hline Lima este & 0,390 & 0,430 & 0,253 & 0,517 & 0,371 & $0,3 \%$ & $45,6 \%$ & $54,1 \%$ \\
\hline Lima sur & 0,382 & 0,434 & 0,279 & 0,496 & 0,392 & $0,7 \%$ & $47,4 \%$ & $52,0 \%$ \\
\hline Balnearios & 0,361 & 0,466 & 0,343 & 0,704 & 0,201 & $0,0 \%$ & $42,4 \%$ & $57,6 \%$ \\
\hline Callao & 0,483 & 0,473 & 0,360 & 0,586 & 0,413 & $0,0 \%$ & $52,4 \%$ & $47,6 \%$ \\
\hline
\end{tabular}

Asimismo, los coeficientes de correlación intraclase (CCI), también relacionados con la similitud de la población que atiende cada tipo de escuela, son más altos para las escuelas no estatales. En primaria, los CCI muestran que la variabilidad del índice socioeconómico de los estudiantes que podría explicarse por las diferencias entre escuelas es $26,4 \%$ en escuelas estatales y $42,2 \%$ en escuelas no estatales. Los valores de este indicador suelen disminuir en secundaria, donde el CCI es 22,7\% para escuelas estatales y 30,5\% para escuelas no estatales. Por lo tanto, en este último tipo de escuelas, existe una mayor probabilidad de que coincidan estudiantes de similar NSE, sobre todo en primaria. Sin embargo, cuando se tiene en cuenta lo sucedido en los distintos tipos de escuelas privadas, los valores que toma el CCI son extrañamente bajos, especialmente para las escuelas de alto y muy alto costo (menores al $10 \%$ tanto en primaria como en secundaria). Aunque este resultado pareciera contraintuitivo, es bastante probable que este indicador haya perdido su poder discriminante al hacer un análisis desagregado de las escuelas por su costo de pensión, puesto que las escuelas de alto y muy alto costo son a la vez similares entre sí, y atienden a una población muy homogénea en términos socioeconómicos. Si bien se requiere investigar con mayor profundidad este fenómeno, esta es una 
de las razones por las que es esencial analizar una serie de indicadores complementarios y fuentes de información que contextualicen los datos numéricos.

En relación con la dimensión de exposición de la segregación escolar en Lima Metropolitana, el índice de aislamiento evidencia que un estudiante de primaria, de escuela estatal y de los NSE más bajos tendría casi $60 \%$ de probabilidad de encontrarse con otro estudiante de su mismo estatus socioeconómico, mientras que, en secundaria, seria de casi 55\%. Dicha probabilidad bajaría a $39,9 \%$ y $31,6 \%$ para primaria y secundaria, respectivamente, en el caso de las escuelas no estatales. En este último tipo de escuelas, la probabilidad de encontrarse con pares de un mismo grupo socioeconómico se eleva a $67 \%$ en primaria y $59 \%$ en secundaria cuando se analiza a los estudiantes de NSE alto. Contrariamente, dicha probabilidad desciende a $26,6 \%$ y $24,2 \%$ en el caso de las escuelas estatales del nivel primario y secundario, respectivamente.

$\mathrm{Al}$ igual que en los casos anteriores, los valores del índice de aislamiento no son homogéneos para los distintos tipos de escuelas privadas. Tanto en primaria como en secundaria, este índice toma valores bastante extremos en las escuelas de medio, alto y muy alto costo, lo que muestra una clara asociación con la composición socioeconómica de la población que atienden. Por ejemplo, en ambos niveles educativos, un estudiante perteneciente a los NSE más bajos tendría menos del 5\% de probabilidad de coincidir con pares de su mismo estatus socioeconómico en las escuelas de alto y muy alto costo, mientras que dicha probabilidad sería de poco más del $15 \%$ en aquellas de medio costo. En el caso de los estudiantes de NSE alto, en primaria, tal probabilidad se eleva a más del 70\% en las escuelas de medio costo, y sobrepasa el $95 \%$ en aquellas de alto y muy alto costo, mientras que, en secundaria, es de 60,2\%; 86,5\%; y $92,2 \%$ en las escuelas de medio, alto y muy alto costo, respectivamente. En cambio, en las escuelas privadas de bajo costo, donde sí existe mayor presencia de estudiantes de distintos NSE, los valores del índice de aislamiento son más moderados. En este caso, la probabilidad de encontrarse con otros estudiantes del mismo grupo socioeconómico, sean de los NSE más bajos o de NSE alto, oscila entre $33,7 \%$ y $42,9 \%$ para primaria y secundaria.

La información brindada por estos índices se corrobora al analizar la caracterización de segregación y la variabilidad del índice socioeconómico al interior de las escuelas de Lima Metropolitana. Del total de escuelas estatales, alrededor de la tercera parte, en primaria, y la cuarta parte, en secundaria, se encuentra en una situación de segregación con una población homogénea. Dicha cifra asciende a poco más de las dos terceras partes en el caso de las escuelas no estatales de ambos niveles educativos. Es más, algunas escuelas con este tipo de gestión, sobre todo aquellas de alto y muy alto costo, poseen una población muy homogénea, puesto que casi la totalidad de sus estudiantes tiene un NSE alto. Además, en promedio, la desviación estándar del índice socioeconómico de los estudiantes de primaria y secundaria es más alta en las escuelas estatales, lo que evidencia una mayor heterogeneidad en su composición escolar. Si 106 I bien las escuelas no estatales de bajo y medio costo poseen cierta variabilidad 
socioeconómica en la población que atienden, esta es muy reducida en aquellas de alto y muy alto costo, especialmente en el caso de primaria.

Para comprender la dinámica de segregación urbana en Lima Metropolitana, también es importante analizar lo sucedido en sus zonas geográficas y en los distritos que las componen. En términos generales, los índices relacionados con la dimensión de similitud de la segregación tienden a valores más elevados en las zonas geográficas con distritos que concentran una mayor proporción de estudiantes de NSE alto.

Si bien todas las zonas geográficas presentan al menos valores moderados en el índice de disimilitud, Lima "moderna" es la única donde dicho índice alcanza valores altos, sobre todo en escuelas de primaria. En este nivel educativo, para conseguir una distribución socioeconómica uniforme, aproximadamente 7 de cada 10 estudiantes de los NSE más bajos tendrían que trasladarse a escuelas con mayor estatus socioeconómico en casi todos los distritos donde más de las dos terceras partes de su composición escolar es de NSE alto (La Molina, Santiago de Surco, Miraflores, San Isidro, San Miguel, San Borja y Jesús María). En el caso de secundaria, es en estos mismos distritos (excepto San Miguel y Jesús María, e incluido Pueblo Libre) donde más del 50\% de los estudiantes de los NSE más bajos deberían desplazarse a escuelas de mayor estatus socioeconómico para alcanzar una distribución proporcional entre todas sus instituciones educativas.

Más allá de lo sucedido en algunos distritos de Lima "moderna", es importante notar dos aspectos transversales: 1) el índice de disimilitud toma valores de moderados a altos en prácticamente todos los distritos de Lima Metropolitana; y 2) los valores de dicho índice suelen mantenerse o incrementarse en las demás zonas geográficas de Lima Metropolitana cuando se toma como referencia a los estudiantes de NSE alto que deberían trasladarse a escuelas de distinto estatus socioeconómico. Aunque estos hallazgos aún deben complementarse con los demás indicadores considerados en este estudio, se evidencia un fuerte grado de segregación en todas las zonas del centro poblado urbano más grande del país. En general, los estudiantes de distintos NSE no se encuentran representados proporcionalmente en la mayoría de las escuelas, conformando grupos de instituciones que atienden a poblaciones desfavorecidas.

Por su parte, los valores del CCI también suelen ser más elevados en primaria en comparación con secundaria en casi todas las zonas geográficas analizadas, excepto en Balnearios. Lima "moderna" y Callao son las zonas que presentan el CCI más alto en primaria y secundaria, respectivamente. En el caso de primaria, el CCI supera el 50\% en distritos como Santiago de Surco, La Molina y Miraflores; es decir, más de la mitad de la variabilidad del índice socioeconómico de los estudiantes se debería a diferencias entre escuelas. No es casualidad, entonces, que dichos distritos presenten los niveles más altos de segregación, focalizada en estudiantes de NSE alto. En secundaria no se aprecia una tendencia tan clara entre el CCI y la distribución socioeconómica de los distritos. Por ejemplo, los distritos del Callao, analizados individualmente, no 
son precisamente los que muestran los valores más altos de CCI. Más bien, el elevado valor de este índice como zona geográfica indicaría que entre sus distritos hay una composición socioeconómica muy dispar, y ello se reflejaría en la existencia de escuelas diferentes entre sí, pero similares internamente en cuanto a la población a la que atienden. Un escenario curioso se presenta en el distrito de Santa Rosa, perteneciente a la zona geográfica de Balnearios. A pesar de que es un distrito con una cantidad relativamente pequeña de escuelas, su elevado CCI tanto en primaria $(50,8 \%)$ como en secundaria $(60,4 \%)$ reflejaría sobre todo la concentración de estudiantes de los NSE más bajos. Asimismo, este es el único distrito que no pertenece a Lima "moderna" o Callao en el que al menos la quinta parte de sus escuelas de primaria se encuentra segregada con una población muy homogénea o donde más del $70 \%$ de sus escuelas de secundaria está en condición de segregación con una población homogénea.

En cuanto a la dimensión de exposición de la segregación escolar, se aprecia una clara relación entre los valores del índice de aislamiento y el NSE de los estudiantes de las distintas zonas geográficas analizadas. Esta tendencia es bastante similar en primaria y secundaria. Por un lado, los estudiantes de los NSE más bajos que tienen una mayor probabilidad de coincidir con pares de su mismo estatus socioeconómico se ubican en escuelas de los distritos periféricos de Lima Metropolitana. Como era de esperarse, dicha probabilidad es bastante alta en distritos con gran parte de población en condición de pobreza, ubicados principalmente en Balnearios (Santa Rosa, Ancón, Pucusana, Punta Negra, Punta Hermosa y San Bartolo); Callao (Ventanilla y Mi Perú); Lima Este (Lurigancho y Cieneguilla); Lima Sur (Pachacámac y Lurín); y Lima Norte (Puente Piedra y Carabayllo). Por otro lado, cuando se tiene en cuenta a los estudiantes de NSE alto, existe una probabilidad de al menos $75 \%$ en primaria y $60 \%$ en secundaria de encontrarse con otros estudiantes del mismo grupo socioeconómico en casi todos de los distritos de Lima "moderna". Aunque en menor grado, esa probabilidad sigue siendo considerable en escuelas de los distritos de Lima Centro, en ciertos distritos del Callao (La Perla, Bellavista y La Punta), así como en algunos distritos periféricos con menos de la cuarta parte de su población en condición de pobreza (Chorrillos y Los Olivos).

Tal como ocurrió con los índices de similitud, son precisamente este grupo de distritos con altos valores en el índice de aislamiento los que suelen tener una mayor proporción de escuelas con población muy homogénea, sobre todo, en primaria. Esta composición escolar extremadamente uniforme, en este caso de estudiantes de NSE alto, se concentra en algunos distritos de Lima "moderna" (San Isidro, Miraflores, Santiago de Surco, La Molina, San Borja y Pueblo Libre). Por lo tanto, no sorprende que la desviación estándar del índice socioeconómico de la población que estudia en esos distritos sea menor a la hallada en las escuelas de otros distritos de esa y otras zonas geográficas. Contrariamente, los distritos con una composición escolar más heterogénea, donde más de la mitad de sus escuelas de primaria y secundaria no se encuentran 108 I segregadas por NSE, pertenecen a Balnearios (San Bartolo, Pucusana y Punta 
Negra); Lima Este (Cieneguilla y San Juan de Lurigancho); Lima Sur (Lurín y Pachacámac); Lima Norte (Puente Piedra); y Callao (Mi Perú y Ventanilla). No obstante, es preocupante que en todos los distritos analizados al menos la tercera parte de las escuelas de primaria y la cuarta parte de las de secundaria se encuentren en condición de segregación.

\section{Discusión}

Los resultados de este estudio evidencian que las escuelas de Lima Metropolitana presentan un alto nivel de segregación socioeconómica. No obstante, este hallazgo no llega a ser una sorpresa, dado que, en los últimos años, diversas investigaciones señalan a Perú como el país que presenta las condiciones de segregación educativa más altas de la región (Benavides et al., 2014; Krüger, 2019; Murillo, 2016; Murillo y Martínez-Garrido, 2017; Murillo et al., 2018; OCDE, 2019; Vazquez, 2016).

La información que se ha presentado en este estudio aborda las diversas formas en que la segregación se puede materializar dentro de las escuelas en Lima Metropolitana. Además, complementa las estimaciones realizadas por Murillo y Carrillo (2020a, 2020b). Sin embargo, es solo una primera mirada descriptiva, necesaria para la estimación de la segregación socioeconómica en distintos estratos y categorías relevantes, pero que requiere ser profundizada para comprender mejor algunos hallazgos de la investigación.

En primer lugar, el hecho de que la incidencia de segregación en primaria sea mayor que en secundaria podría deberse a la desigual cantidad de escuelas primarias y secundarias en la capital. Aquellas de nivel primario representan poco menos del doble del total de escuelas del nivel secundario. Asimismo, la tasa de transición a secundaria se encuentra por encima del $97 \%$. Todo ello propiciaría que los estudiantes coincidan en un menor número de establecimientos, de ahí que se reduzca la segregación socioeconómica dentro de las escuelas.

Otra posible explicación recae en la interacción entre el efecto de la segregación residencial y el proceso de elección de escuela por parte de las familias. Al ser Lima Metropolitana un área segregada, es probable que los estudiantes más pequeños, sobre todo de los NSE más bajos, asistan a las escuelas de sus barrios. En cambio, en secundaria, cuando los estudiantes suelen trasladarse más y de manera más autónoma para ir a la escuela, las familias suelen matricular a los estudiantes en las que consideran "mejores" instituciones, como parte de un mecanismo aspiracional (Cuenca, 2013).

En segundo lugar, los resultados indican que la segregación no es un fenómeno uniforme, pues se manifiesta de manera diferenciada según las distintas categorías analizadas. En tal sentido, depende de las características de la escuela y del contexto en el que se encuentre situada. Por ejemplo, el análisis realizado con la tipología de escuelas no estatales (Minedu, 2018b) revela la existencia de diferencias en la composición socioeconómica del alumnado y, 
por consiguiente, distintos grados de segregación que presenta cada tipo de escuela. Por otro lado, si bien las escuelas estatales muestran cierto grado de heterogeneidad en cuanto a la población que atienden, también es importante seguir explorando las dinámicas diferenciadas al interior de la oferta pública, entre ellas, la discrecionalidad en los procesos de admisión escolar.

En referencia a la segregación en escuelas privadas, la dinámica del mercado educativo comprende un escenario bastante complejo y demanda intervenciones específicas. Por ejemplo, son necesarios programas de fortalecimiento en la gestión y capacitación docente en aquellas escuelas estatales de zonas periurbanas marginales y, además, una mayor regulación en la oferta educativa de las escuelas privadas. Esto, en la ruta de garantizar la igualdad de oportunidades entre todos los estudiantes y construir un sistema educativo más equitativo e inclusivo. Así también, demanda que el Estado tome medidas para limitar la segregación de los estudiantes con mayores recursos con el fin asegurar la equidad y diversidad en las escuelas privadas.

Por último, respecto al análisis por zona geográfica, los indicadores de segregación se muestran especialmente altos en los distritos más privilegiados de la ciudad (Lima "moderna"). Esto puede estar relacionado a la falta de articulación del Estado para responder a la creciente demanda por servicios educativos en la capital. Lima Metropolitana ha presentado un rápido crecimiento poblacional; en efecto, se ha ampliado la periferia de la ciudad y, como consecuencia, se ha incrementado la densidad poblacional en la mayoría de los distritos (Matos Mar, 2012). Por otro lado, el Estado no ha sido efectivo en el objetivo de ampliar la oferta educativa estatal, lo que, a su vez, ha permitido la aparición desregulada de escuelas no estatales denominadas "de bajo costo", que se presentan como solución a esa demanda insatisfecha.

Lo presentado en el estudio señala como uno de los retos educativos con mayor relevancia lograr que las escuelas, sobre todo las estatales, puedan llegar a ser espacios de aprendizaje donde las condiciones socioeconómicas no representen una limitante o ventaja para el desarrollo de aprendizajes en los estudiantes. Si bien esta problemática es compleja de abordar, es importante resaltar los pasos avanzados en materia de calidad docente, como la promulgación de la Ley de Reforma Magisterial y la puesta en marcha de evaluaciones basadas en el mérito. También, se han fortalecido los aprendizajes, y se cuenta con el soporte de los programas de acompañamiento pedagógico y capacitación docente emprendidos por el gobierno central y los gobiernos locales.

La inclusión de la segregación escolar en el Proyecto Educativo Nacional al 2036 (CNE, 2020) constituye un hito importante, puesto que es la primera vez que hay un reconocimiento oficial de esta problemática. Así, se incluye en la agenda de la política educativa. Esto es positivo en tanto aumenta la probabilidad de que se puedan establecer medidas para enfrentarla. Como se señala en el PEN: 
el principal mecanismo para combatir la segregación del sistema educativo y con ello favorecer las experiencias de aprendizaje y que las instituciones educativas sean espacios de encuentro entre nuestras diversidades - con lo que se construye mayores niveles de cohesión social- es fortalecer la educación estatal gratuita. (CNE, 2020, p. 27)

El presente estudio ha intentado aproximarse a la medición de la magnitud de la segregación socioeconómica integrando diferentes métodos y considerando distintas características específicas de las escuelas de Lima Metropolitana. Se espera que los hallazgos brindados contribuyan con la reflexión y el debate en torno a esta problemática, orientando la urgente generación de acciones concretas que conduzcan a resolverla. Es tiempo de construir una sociedad más justa, inclusiva y cohesionada, pensada desde el verdadero rol de la escuela y de la educación básica. 


\section{Referencias bibliográficas}

Alegre, M. A. (2010). Casi-mercados, segregación escolar y desigualdad educativa: una trilogía con final abierto. Educação \& Sociedade, 31(113), 1157-1178.

Allen, R.y Vignoles, A. (2007). What Should an Index of School Segregation Measure? London School of Economics, Centre for the Economics of Education.

Arcidiácono, M., Cruces, G., Gasparini, L., Jaume, D., Serio, M. y Vázquez, E. (2014). La segregación escolar público-privada en América Latina. CEPAL.

Balarin, M. (2016). La privatización por defecto y el surgimiento de las escuelas privadas de bajo costo en el Perú. ¿Cuáles son sus consecuencias? Revista de la Asociación de Sociología de La Educación, 9(2), 181-196.

Balarin, M., Kitmang, J., Ñopo, H. y Rodríguez, M. F. (2018). Mercado privado, consecuencias públicas. Los servicios educativos de provisión privada en el Perú. Grade.

Balarin, M. y Escudero, A. (2019). The Ungoverned Education Market and the Deepening of Socio-Economic School Segregation in Peru. En X. Bonal y C. Bellei (Eds.), Undestanding School Segregation. Patterns, Causes and Consequences of Spatial Inequalities in Education. Bloomsbury Academic.

Bellei, C. (2013). El estudio de la segregación socioeconómica y académica de la educación chilena. Estudios Pedagógicos XXXIX, (1), 325-345.

Benavides, M., León, J. y Etesse, M. (2014). Desigualdades educativas y segregación en el sistema educativo peruano. Una mirada comparativa de las pruebas PISA 2000 y 2009. Grade.

Bonal, X. y Bellei, C. (2019). Introduction: The Renaissance of School Segregation in A Context of Globalization. En X. Bonal y C. Bellei (Eds.), Undestanding School Segregation. Patterns, Causes and Consequences of Spatial Inequalities in Education. Bloomsbury Academic.

Carrillo, S. (2020). La segregación escolar en América Latina ¿Qué se estudia y cómo se investiga? REICE. Revista Iberoamericana sobre Calidad, Eficacia y Cambio en Educación, 18(4), 345-362.

Carrillo, S., Salazar, V. y Leandro, S. (2019). Jóvenes y educación en Lima Metropolitana y Callao. IEP.

Consejo Nacional de Educación [CNE] (2020). Proyecto Educativo Nacional al 2036: el reto de la ciudadanía plena. CNE.

Cuenca, R. (2013). La escuela pública en Lima Metropolitana. ¿Una institución en extinción? Revista Peruana de Investigación Educativa, 5, 73-98.

Cueto, S., León, J. y Miranda, A. (2016). Classroom Composition and its Association with Students' Achievement and Socioemotional Characteristics in Peru. Assessment in Education: Principles, Policy and 112 I Practice, 23(1), 126-148. 
Duncan, O. y Duncan, B. (1955). A Methodological Analysis of Segregation Indexes. American Sociological Review, 20(2), 210-217.

Dupriez, V. (2010). Methods of Grouping Learners at School. Unesco.

Echenique, F. y Fryer, R. (2007). A Measure of Segregation Based on Social Interactions. The Quarterly Journal of Economics, 122(2), 441-485.

Gorard, S. y Taylor, C. (2002). What Is Segregation? A Comparison of Measures in Terms of 'Strong' and 'Weak' Compositional Invariance. Sociology, 36(4), 875-895.

Guadalupe, C., León, J. y Cueto, S. (2013). Charting Progress in Learning Outcomes in Perú Using National Assessments. Background paper prepared for the EFA Global Monitoring Report 2013/4. Teaching and learning: achieving quality for all. Unesco.

Instituto Nacional de Estadística e Informática (2014). Una mirada a Lima Metropolitana. INEI.

Instituto Nacional de Estadística e Informática (2021). Encuesta Nacional de Hogares 2021. https://www.inei.gob.pe/

Ipsos (2018). Perfiles zonales Lima 2018. Ipsos.

James, D. R. y Taeuber, K. E. (1985). Measures of Segregation. Sociological Methodology, 15, 1-32.

Krüger, N. (2019). La segregación por nivel socioeconómico como dimensión de la exclusión educativa: 15 años de evolución en América Latina. Archivos Analiticos de Políticas Educativas, 27(8), 1-34.

León, J. y Collahua, Y. (2016). El efecto del nivel socioeconómico en el rendimiento de los estudiantes peruanos: un balance de los últimos 15 años. En Grade (Ed.), Investigación para el desarrollo en el Perú. Once balances. Grade.

Massey, D. S. y Denton, N. A. (1988). The Dimensions of Residential Segregation. Social forces, 67(2), 281-315.

Matos Mar, J. (2012). Perú: Estado desbordado y sociedad nacional emergente. Universidad Ricardo Palma.

Mikulyuk, A. y Braddock, J. (2016). K-12 School Diversity and Social Cohesion: Evidence in Support of a Compelling State Interest. Education and Urban Society, 1(13), 1-33.

Ministerio de Educación [Minedu] (2016). ¿Cuánto aprenden nuestros estudiantes al término de la educación primaria? Informe de logros de aprendizaje y sus factores asociados en la Evaluación Muestral 2013. Oficina de Medición de la Calidad de los Aprendizajes.

Ministerio de Educación [Minedu] (2018a). Desafíos en la medición y análisis del estatus socioeconómico de los estudiantes peruanos. Oficina de Medición de la Calidad de los Aprendizajes. 
Ministerio de Educación [Minedu] (2018b). Tipología y caracterización de las escuelas privadas en el Perú. Estudios Breves N. ${ }^{\circ}$ 3. Oficina de Medición de la Calidad de los Aprendizajes.

Ministerio de Educación [Minedu] (2021). Estadística de la Calidad Educativa. http://escale.minedu.gob.pe/

Miranda, L. (2008). Factores asociados al rendimiento escolar y sus implicancias para la política educativa del Perú. En M. Benavides (Ed.), Análisis de programas, procesos y resultados educativos en el Perú: contribuciones empiricas para el debate. Grade.

Murillo, F. J. (2016). Midiendo la segregación escolar en América Latina. Un análisis metodológico utilizando el TERCE. REICE. Revista Iberoamericana Sobre Calidad, Eficacia y Cambio en Educación, 14(4), 33-60.

Murillo, F. J. y Carrillo, S. (2020a). Una panorámica de la segregación escolar por nivel socioeconómico en educación primaria en Perú y sus regiones. Argumentos. Revista de Ciencias Sociales, 1(1), 7-31.

Murillo, F. J. y Carrillo, S. (2020b). Segregación escolar por nivel socioeconómico en educación secundaria en Perú y sus regiones. Revista Peruana de Investigación Educativa, 12, 7-32.

Murillo, F. J. y Carrillo, S. (2021). Incidencia de la segregación escolar por nivel socioeconómico en el rendimiento académico. Un estudio desde Perú. Archivos Analíticos de Políticas Educativas, 29(49), 1-24.

Murillo, F. J. y Graña Oliver, R. (2020). Una panorámica de la segregación escolar por nivel socioeconómico en Uruguay. Cuadernos de Investigación Educativa, 11(1), 15-35.

Murillo, F. J. y Martínez-Garrido, C. (2016). Segregación social en las escuelas públicas y privadas en América Latina. Educação \& Sociedade, 38(140), 727-750.

Murillo, F. J. y Martínez-Garrido, C. (2017). Estimación de la magnitud de la segregación escolar en América Latina. Magis. Revista Internacional de Investigación En Educación, 9(19), 11-30.

Murillo, F. J., Duk, C. y Martínez-Garrido, C. (2018). Evolución de la segregación socioeconómica de las escuelas de América Latina. Estudios Pedagógicos, 44(1), 157-179.

Murillo, F. J., Martínez-Garrido, C. y Graña Oliver, R. (2020). Escuelas públicas para pobres, escuelas privadas para ricos: relación entre educación privada y segregación escolar de carácter socio-económico en América Latina. Revista Científica RUNAE, 5, 11-22.

OECD. (2019). PISA 2018 Results (Volume II): Where All Students Can Succeed. OECD Publishing.

Pereyra, O. (2006). Forma urbana y segregación residencial en Lima. Debates en Sociología, 31, 69-106. 
Talavera, G. (2019). Segregación residencial en Lima Metropolitana: autocorrelación espacial y aglomeración en la ciudad (Tesis de Maestría). Universidad Autónoma de Barcelona.

Valenzuela, J. P., Bellei, C. y De los Ríos, D. (2010) Segregación Escolar en Chile. En S. Martinic y G. Elacqua (Eds.), ¿Fin de ciclo? Cambios en la gobernanza del sistema educativo. Orealc/Unesco - Pontificia Universidad Católica de Chile.

Vazquez, E. (2016). Segregación escolar por nivel socioeconómico. Midiendo el fenómeno y explorando sus determinantes. Económica, 62, 121-184.

Wilkes, R. y Iceland, J. (2004). Hypersegregation in the twenty-first century. Demography, 41(1), 23-36. 


\section{Anexos}

\section{Anexo A: Distribución socioeconómica en las escuelas de Lima Metropolitana}

Tabla A1

Distribución socioeconómica de las escuelas de $4 .^{\circ}$ grado de primaria, según tipo de gestión, costo de pensión, zona geográfica y distrito

\begin{tabular}{|c|c|c|c|c|c|c|}
\hline \multirow{2}{*}{ Estrato } & \multirow{2}{*}{$\mathrm{N}$} & \multicolumn{4}{|c|}{ NSE } & \multirow{2}{*}{$\mathrm{DE}$} \\
\hline & & Muy bajo & Bajo & Medio & Alto & \\
\hline Estatal & 1055 & $17,6 \%$ & $32,7 \%$ & $37,5 \%$ & $12,1 \%$ & 0,792 \\
\hline No estatal & 3344 & $3,9 \%$ & $13,8 \%$ & $36,1 \%$ & $46,3 \%$ & 0,663 \\
\hline Muy alto costo & 31 & $0,0 \%$ & $0,0 \%$ & $1,8 \%$ & $98,1 \%$ & 0,106 \\
\hline Alto costo & 69 & $0,0 \%$ & $0,2 \%$ & $3,3 \%$ & $96,5 \%$ & 0,165 \\
\hline Medio costo & 880 & $0,7 \%$ & $4,8 \%$ & $28,0 \%$ & $66,4 \%$ & 0,544 \\
\hline Bajo costo & 2350 & $6,5 \%$ & $21,8 \%$ & $46,6 \%$ & $25,0 \%$ & 0,730 \\
\hline Lima norte & 1203 & $10,1 \%$ & $23,9 \%$ & $40,7 \%$ & $25,3 \%$ & 0,718 \\
\hline Lima centro & 326 & $5,8 \%$ & $17,7 \%$ & $38,6 \%$ & $37,9 \%$ & 0,683 \\
\hline Lima moderna & 435 & $2,2 \%$ & $6,7 \%$ & $20,6 \%$ & $70,5 \%$ & 0,438 \\
\hline Lima este & 1087 & $13,9 \%$ & $27,6 \%$ & $38,9 \%$ & $19,5 \%$ & 0,749 \\
\hline Lima sur & 823 & $12.8 \%$ & $26,8 \%$ & $38,3 \%$ & $22,1 \%$ & 0,735 \\
\hline Balnearios & 69 & $36,1 \%$ & $30,7 \%$ & $26,5 \%$ & $6,7 \%$ & 0,745 \\
\hline Callao & 456 & $12,0 \%$ & $27,4 \%$ & $37,4 \%$ & $23,2 \%$ & 0,674 \\
\hline Ancón & 33 & $35,8 \%$ & $31,8 \%$ & $26,3 \%$ & $6,1 \%$ & 0,783 \\
\hline Ate & 312 & $14,2 \%$ & $28,3 \%$ & $37,5 \%$ & $20,0 \%$ & 0,744 \\
\hline Barranco & 23 & $2,9 \%$ & $13,5 \%$ & $38,5 \%$ & $45,1 \%$ & 0,635 \\
\hline Breña & 40 & $1,7 \%$ & $7,7 \%$ & $30,1 \%$ & $60,4 \%$ & 0,621 \\
\hline Carabayllo & 156 & $18,7 \%$ & $30,2 \%$ & $35,0 \%$ & $16,2 \%$ & 0,751 \\
\hline Chaclacayo & 29 & $7,5 \%$ & $19,1 \%$ & $41,7 \%$ & $31,7 \%$ & 0,723 \\
\hline Chorrillos & 143 & $6,0 \%$ & $19,1 \%$ & $35,5 \%$ & $39,4 \%$ & 0,682 \\
\hline Cieneguilla & 13 & $19,0 \%$ & $37,1 \%$ & $36,5 \%$ & $7,4 \%$ & 0,768 \\
\hline Comas & 240 & $9,2 \%$ & $22,1 \%$ & $42,9 \%$ & $25,8 \%$ & 0,739 \\
\hline El Agustino & 70 & $8,2 \%$ & $28,2 \%$ & $45,2 \%$ & $18,4 \%$ & 0,732 \\
\hline Independencia & 77 & $12,1 \%$ & $27,1 \%$ & $42,9 \%$ & $17,9 \%$ & 0,740 \\
\hline Jesús María & 24 & $0,5 \%$ & $5,2 \%$ & $21,2 \%$ & $73,2 \%$ & 0,423 \\
\hline La Molina & 62 & $5,8 \%$ & $9.2 \%$ & $17,4 \%$ & $67,6 \%$ & 0,385 \\
\hline La Victoria & 69 & $7,0 \%$ & $22,5 \%$ & $38,0 \%$ & $32,5 \%$ & 0,690 \\
\hline Lima & 123 & $4,5 \%$ & $16,8 \%$ & $39,8 \%$ & $38,9 \%$ & 0,680 \\
\hline Lince & 18 & $2,0 \%$ & $10,7 \%$ & $32,1 \%$ & $55,2 \%$ & 0,523 \\
\hline Los Olivos & 189 & $4,4 \%$ & $18,5 \%$ & $39,4 \%$ & $37,7 \%$ & 0,658 \\
\hline Lurigancho & 123 & $23,8 \%$ & $29,4 \%$ & $33,0 \%$ & $13,9 \%$ & 0,761 \\
\hline Lurin & 47 & $24,3 \%$ & $31,7 \%$ & $32,5 \%$ & $11,5 \%$ & 0,819 \\
\hline Magdalena del Mar & 22 & $2,1 \%$ & $7,4 \%$ & $25,7 \%$ & $64,8 \%$ & 0,536 \\
\hline Miraflores & 27 & $0,9 \%$ & $4,2 \%$ & $13,5 \%$ & $81,4 \%$ & 0,340 \\
\hline Pachacámac & 53 & $32,6 \%$ & $31,4 \%$ & $24,8 \%$ & $11,2 \%$ & 0,804 \\
\hline Pucusana & 9 & $40,4 \%$ & $30,6 \%$ & $23,0 \%$ & $6,0 \%$ & 0,871 \\
\hline Pueblo Libre & 28 & $0,9 \%$ & $5,5 \%$ & $25.8 \%$ & $67,9 \%$ & 0,454 \\
\hline Puente Piedra & 179 & $18,8 \%$ & $34,7 \%$ & $35,2 \%$ & $11,3 \%$ & 0,778 \\
\hline Punta Hermosa & 1 & $54,3 \%$ & $14,3 \%$ & $31,4 \%$ & $0,0 \%$ & 0,910 \\
\hline Punta Negra & 7 & $15,5 \%$ & $45,6 \%$ & $31,1 \%$ & $7,8 \%$ & 0,533 \\
\hline Rímac & 69 & $8,4 \%$ & $18,6 \%$ & $40,8 \%$ & $32,2 \%$ & 0,691 \\
\hline San Bartolo & 4 & $28,2 \%$ & $28,2 \%$ & $35,5 \%$ & $8.2 \%$ & 0,597 \\
\hline San Borja & 24 & $1,6 \%$ & $4.8 \%$ & $17,5 \%$ & $76,1 \%$ & 0,389 \\
\hline San Isidro & 19 & $0,3 \%$ & $3,0 \%$ & $9,2 \%$ & $87,5 \%$ & 0,278 \\
\hline San Juan de Lurigancho & 449 & $14,3 \%$ & $27,6 \%$ & $38,4 \%$ & $19,7 \%$ & 0,763 \\
\hline San Juan de Miraflores & 177 & $10.1 \%$ & $24,5 \%$ & $41.1 \%$ & $24,3 \%$ & 0,715 \\
\hline San Luis & 25 & $7,7 \%$ & $22,1 \%$ & $41,5 \%$ & $28,7 \%$ & 0,756 \\
\hline San Martín de Porres & 362 & $4,4 \%$ & $18,3 \%$ & $45,5 \%$ & $31,8 \%$ & 0,686 \\
\hline San Miguel & 63 & $1,0 \%$ & $4,2 \%$ & $18,4 \%$ & $76,4 \%$ & 0,494 \\
\hline Santa Anita & 91 & $5,6 \%$ & $24,6 \%$ & $47,1 \%$ & $22,7 \%$ & 0,706 \\
\hline Santa María del Mar & 1 & $0,0 \%$ & $0,0 \%$ & $60,0 \%$ & $40,0 \%$ & 0,548 \\
\hline Santa Rosa & 14 & $45,2 \%$ & $24,0 \%$ & $22,2 \%$ & $8,6 \%$ & 0,734 \\
\hline Santiago de Surco & 102 & $1,7 \%$ & $6,1 \%$ & $19,0 \%$ & $73,2 \%$ & 0,384 \\
\hline Surquillo & 23 & $3,8 \%$ & $10,0 \%$ & $31,1 \%$ & $55,1 \%$ & 0,593 \\
\hline Villa el Salvador & 196 & $8,6 \%$ & $30,3 \%$ & $43,4 \%$ & $17,6 \%$ & 0,731 \\
\hline Villa Maria del Triunfo & 207 & $16,1 \%$ & $29,6 \%$ & $38,8 \%$ & $15,5 \%$ & 0,756 \\
\hline Bellavista & 50 & $1,1 \%$ & $7,9 \%$ & $34,5 \%$ & $56,5 \%$ & 0,604 \\
\hline Callao & 188 & $4,2 \%$ & $22,3 \%$ & $46.9 \%$ & $26.6 \%$ & 0,662 \\
\hline Carmen de la Legua & 20 & $2,8 \%$ & $23,7 \%$ & $44.6 \%$ & $29,0 \%$ & 0,634 \\
\hline La Perla & 26 & $1,9 \%$ & $14,0 \%$ & $35,0 \%$ & $49,2 \%$ & 0,511 \\
\hline La Punta & 3 & $3,8 \%$ & $19,2 \%$ & $36,5 \%$ & $40,4 \%$ & 0,572 \\
\hline Mi Perú & 22 & $17,4 \%$ & $41,7 \%$ & $34,5 \%$ & $6,4 \%$ & 0,741 \\
\hline Ventanilla & 147 & $24,5 \%$ & $37,7 \%$ & $28,2 \%$ & $9,6 \%$ & 0,738 \\
\hline
\end{tabular}


Tabla A2

Distribución socioeconómica de las escuelas de $2 .^{\circ}$ grado de secundaria, según tipo de gestión, costo de pensión, zona geográfica y distrito

\begin{tabular}{|c|c|c|c|c|c|c|}
\hline \multirow{2}{*}{ Estrato } & \multirow{2}{*}{$\mathrm{N}$} & \multicolumn{4}{|c|}{ NSE } & \multirow{2}{*}{$\mathrm{DE}$} \\
\hline & & Muy bajo & Bajo & Medio & Alto & \\
\hline Estatal & 758 & $14,3 \%$ & $32,0 \%$ & $38,9 \%$ & $14,8 \%$ & 0,814 \\
\hline No cstatal & 2183 & $2,7 \%$ & $13,1 \%$ & $38,9 \%$ & $45,2 \%$ & 0,699 \\
\hline Muy alto costo & 30 & $0,2 \%$ & $0,8 \%$ & $7,2 \%$ & $91,8 \%$ & 0,332 \\
\hline Alto costo & 70 & $0,2 \%$ & $1,3 \%$ & $12,8 \%$ & $85,7 \%$ & 0,428 \\
\hline Medio costo & 855 & $1,1 \%$ & $8,0 \%$ & $36,9 \%$ & $54,0 \%$ & 0,651 \\
\hline Bajo costo & 1212 & $5,3 \%$ & $21,9 \%$ & $47,5 \%$ & $25,3 \%$ & 0,758 \\
\hline Lima norte & 791 & $8,2 \%$ & $24,0 \%$ & $41,7 \%$ & $26,0 \%$ & 0,739 \\
\hline Lima centro & 233 & $5,4 \%$ & $19,8 \%$ & $41,8 \%$ & $33.0 \%$ & 0,726 \\
\hline Lima moderna & 372 & $2,1 \%$ & $9,9 \%$ & $28,8 \%$ & $59,2 \%$ & 0,589 \\
\hline Lima este & 706 & $11,7 \%$ & $27,2 \%$ & $40,2 \%$ & $20,9 \%$ & 0,766 \\
\hline Lima sur & 517 & $10,7 \%$ & $26,6 \%$ & $40,7 \%$ & $22,0 \%$ & 0,761 \\
\hline Balnearios & 36 & $27,9 \%$ & $35,3 \%$ & $27,6 \%$ & $9,2 \%$ & 0,758 \\
\hline Callao & 286 & $12,5 \%$ & $27,5 \%$ & $37,8 \%$ & $22,2 \%$ & 0,734 \\
\hline Ancón & 15 & $26,8 \%$ & $36,3 \%$ & $29,4 \%$ & $7,5 \%$ & 0,752 \\
\hline Ate & 187 & $11,8 \%$ & $28,3 \%$ & $38,2 \%$ & $21,7 \%$ & 0,768 \\
\hline Barranco & 20 & $2,4 \%$ & $18,6 \%$ & $40,3 \%$ & $38,7 \%$ & 0,655 \\
\hline Breña & 35 & $1,8 \%$ & $12,3 \%$ & $38,4 \%$ & $47,5 \%$ & 0,690 \\
\hline Carabayllo & 101 & $14,3 \%$ & $30,2 \%$ & $36,8 \%$ & $18,7 \%$ & 0,758 \\
\hline Chaclacayo & 22 & $7,8 \%$ & $24,5 \%$ & $43,5 \%$ & $24,2 \%$ & 0,683 \\
\hline Chorrillos & 92 & $6,0 \%$ & $18,9 \%$ & $37,8 \%$ & $37,3 \%$ & 0,705 \\
\hline Cieneguilla & 10 & $14,7 \%$ & $33,6 \%$ & $35,9 \%$ & $15,8 \%$ & 0,842 \\
\hline Comas & 163 & $7,4 \%$ & $21,6 \%$ & $43.9 \%$ & $27,1 \%$ & 0,741 \\
\hline El Agustino & 45 & $8,3 \%$ & $27,7 \%$ & $45.3 \%$ & $18,6 \%$ & 0,766 \\
\hline Independencia & 53 & $10,2 \%$ & $27,3 \%$ & $44,4 \%$ & $18,2 \%$ & 0,791 \\
\hline Jesús María & 25 & $1,8 \%$ & $9,7 \%$ & $35,2 \%$ & $53,2 \%$ & 0,665 \\
\hline La Molina & 57 & $3,9 \%$ & $10,3 \%$ & $23,4 \%$ & $62,5 \%$ & 0,546 \\
\hline La Victoria & 45 & $8,1 \%$ & $23,0 \%$ & $42,3 \%$ & $26,5 \%$ & 0,739 \\
\hline Lima & 84 & $4,3 \%$ & $19,3 \%$ & $42,2 \%$ & $34,2 \%$ & 0,728 \\
\hline Lince & 19 & $1,6 \%$ & $10,0 \%$ & $38,6 \%$ & $49.8 \%$ & 0,633 \\
\hline Los Olivos & 134 & $3,4 \%$ & $18,6 \%$ & $41,7 \%$ & $36,2 \%$ & 0,705 \\
\hline Lurigancho & 77 & $19.9 \%$ & $30,4 \%$ & $33,6 \%$ & $16.2 \%$ & 0,775 \\
\hline Lurin & 23 & $18,3 \%$ & $34,8 \%$ & $32,3 \%$ & $14.6 \%$ & 0,849 \\
\hline Magdalena del Mar & 16 & $2,4 \%$ & $12,2 \%$ & $36,1 \%$ & $49,2 \%$ & 0,627 \\
\hline Miraflores & 27 & $2,4 \%$ & $10,5 \%$ & $27,5 \%$ & $59,6 \%$ & 0,567 \\
\hline Pachacámac & 38 & $27,2 \%$ & $36,9 \%$ & $26,7 \%$ & $9,2 \%$ & 0,807 \\
\hline Pucusana & 6 & $34,0 \%$ & $38,3 \%$ & $23,0 \%$ & $4,8 \%$ & 0,772 \\
\hline Pueblo Libre & 20 & $0,7 \%$ & $6,4 \%$ & $25,1 \%$ & $67,8 \%$ & 0,600 \\
\hline Puente Piedra & 121 & $15,8 \%$ & $36,3 \%$ & $35,9 \%$ & $12,1 \%$ & 0,796 \\
\hline Punta Hermosa & 1 & $38.6 \%$ & $32,9 \%$ & $22,9 \%$ & $5.7 \%$ & 0,924 \\
\hline Punta Negra & 5 & $22,3 \%$ & $34,7 \%$ & $29.8 \%$ & $13.2 \%$ & 0,845 \\
\hline Rímac & 48 & $7,3 \%$ & $23,2 \%$ & $43,2 \%$ & $26,3 \%$ & 0,734 \\
\hline San Bartolo & 1 & $17,5 \%$ & $36,4 \%$ & $28,7 \%$ & $17,5 \%$ & 0,977 \\
\hline San Borja & 19 & $1,6 \%$ & $7,4 \%$ & $22,9 \%$ & $68,0 \%$ & 0,543 \\
\hline San Isidro & 17 & $0,6 \%$ & $4,8 \%$ & $21,1 \%$ & $73,6 \%$ & 0,410 \\
\hline San Juan de Lurigancho & 302 & $11,9 \%$ & $26,9 \%$ & $40,7 \%$ & $20,6 \%$ & 0,774 \\
\hline San Juan de Miraflores & 108 & $9,3 \%$ & $23,4 \%$ & $42,4 \%$ & $24,9 \%$ & 0,771 \\
\hline San Luis & 21 & $6,4 \%$ & $21,1 \%$ & $42,2 \%$ & $30,3 \%$ & 0,737 \\
\hline San Martín de Porres & 219 & $4,1 \%$ & $18,6 \%$ & $45,0 \%$ & $32,2 \%$ & 0,705 \\
\hline San Miguel & 56 & $1,3 \%$ & $8,2 \%$ & $30,2 \%$ & $60,3 \%$ & 0,649 \\
\hline Santa Anita & 63 & $5,2 \%$ & $22,8 \%$ & $45,8 \%$ & $26,2 \%$ & 0,727 \\
\hline Santa Rosa & 8 & $34,4 \%$ & $27,3 \%$ & $23,0 \%$ & $15,3 \%$ & 0,655 \\
\hline Santiago de Surco & 79 & $1.8 \%$ & $8,3 \%$ & $24,9 \%$ & $65,0 \%$ & 0,564 \\
\hline Surquillo & 17 & $3,5 \%$ & $16,7 \%$ & $38,4 \%$ & $41,4 \%$ & 0,631 \\
\hline Villa el Salvador & 135 & $7,4 \%$ & $28,0 \%$ & $46,3 \%$ & $18,4 \%$ & 0,744 \\
\hline Villa María del Triunfo & 121 & $12,6 \%$ & $29,5 \%$ & $41,8 \%$ & $16,1 \%$ & 0,781 \\
\hline Bellavista & 35 & $2,3 \%$ & $15,8 \%$ & $42,1 \%$ & $39,8 \%$ & 0,683 \\
\hline Callao & 117 & $4,7 \%$ & $22,9 \%$ & $45,4 \%$ & $27,0 \%$ & 0,708 \\
\hline Carmen de la Legua & 9 & $3,7 \%$ & $19,4 \%$ & $50,8 \%$ & $26,1 \%$ & 0,726 \\
\hline La Perla & 19 & $1,8 \%$ & $11,7 \%$ & $37,0 \%$ & $49,5 \%$ & 0,701 \\
\hline La Punta & 2 & $0,0 \%$ & $6,5 \%$ & $41,9 \%$ & $51,6 \%$ & 0,603 \\
\hline Mi Perú & 11 & $18,7 \%$ & $39,2 \%$ & $34,2 \%$ & $7,9 \%$ & 0,822 \\
\hline Ventanilla & 93 & $25,6 \%$ & $37,3 \%$ & $27,1 \%$ & $10,1 \%$ & 0,783 \\
\hline
\end{tabular}




\section{Anexo B: Indicadores de segregación en los distritos de Lima Metropolitana}

Tabla B1

Indicadores de segregación en $4 .^{\circ}$ grado de primaria, según distrito

\begin{tabular}{|c|c|c|c|c|c|c|c|c|}
\hline \multirow{2}{*}{ Distrito } & \multicolumn{2}{|c|}{ Disimilitud } & \multirow{2}{*}{$1 \mathrm{CC}$} & \multicolumn{2}{|c|}{ Aislamiento } & \multicolumn{3}{|c|}{ Grado de segregación } \\
\hline & $\mathrm{MB}+\mathrm{B}$ & Alto & & $\mathrm{MB}+\mathrm{B}$ & Alto & Muy hom & Hom & Het \\
\hline Ancón & 0,434 & 0,491 & 0,284 & 0,758 & 0,180 & $0,0 \%$ & $60,9 \%$ & $39,1 \%$ \\
\hline Ate & 0.449 & 0.579 & 0,340 & 0,577 & 0,498 & $1,6 \%$ & $53,9 \%$ & $44.4 \%$ \\
\hline Barranco & 0,480 & 0,522 & 0,379 & 0,295 & 0,631 & $14,3 \%$ & $66,7 \%$ & $19.0 \%$ \\
\hline Breña & 0,513 & 0,484 & 0,254 & 0,203 & 0,714 & $8,6 \%$ & $68,6 \%$ & $22,9 \%$ \\
\hline Carabayllo & 0,480 & 0,550 & 0,359 & 0,643 & 0,396 & $0,0 \%$ & $54,7 \%$ & $45,3 \%$ \\
\hline Chaclacayo & 0,463 & 0,561 & 0,300 & 0,418 & 0,547 & $4,5 \%$ & $63,6 \%$ & $31,8 \%$ \\
\hline Chorrillos & 0,522 & 0,601 & 0,404 & 0,444 & 0,653 & $6,9 \%$ & $59,5 \%$ & $33,6 \%$ \\
\hline Cieneguilla & 0,320 & 0,605 & 0,238 & 0,634 & 0,253 & $0,0 \%$ & $41,7 \%$ & $58,3 \%$ \\
\hline Comas & 0,390 & 0,452 & 0,266 & 0,448 & 0,430 & $0,5 \%$ & $54,9 \%$ & $44,5 \%$ \\
\hline El Agustino & 0,371 & 0,392 & 0,253 & 0,481 & 0,319 & $0,0 \%$ & $50,0 \%$ & $50,0 \%$ \\
\hline Independencia & 0,363 & 0,462 & 0,215 & 0,500 & 0,334 & $0,0 \%$ & $51,7 \%$ & $48,3 \%$ \\
\hline Jesús Maria & 0,674 & 0,529 & 0,279 & 0,181 & 0,813 & $21,1 \%$ & $73,7 \%$ & $5,3 \%$ \\
\hline La Molina & 0,779 & 0,768 & 0,540 & 0,531 & 0,881 & $49,0 \%$ & $35,3 \%$ & $15,7 \%$ \\
\hline La Victoria & 0,469 & 0,483 & 0,384 & 0,486 & 0,530 & $1,7 \%$ & $56,9 \%$ & $41,4 \%$ \\
\hline Lima & 0,458 & 0,508 & 0,362 & 0,371 & 0,592 & $4,8 \%$ & $60,6 \%$ & $34,6 \%$ \\
\hline Lince & 0,477 & 0,563 & 0,316 & 0,230 & 0,709 & $7,1 \%$ & $71,4 \%$ & $21,4 \%$ \\
\hline Las Olivos & 0,475 & 0,533 & 0,291 & 0,388 & 0,590 & $4,0 \%$ & $72,0 \%$ & $24,0 \%$ \\
\hline Lurigancho & 0,502 & 0,614 & 0,409 & 0,687 & 0,416 & $1,0 \%$ & $49,5 \%$ & $49,5 \%$ \\
\hline Lurín & 0,399 & 0,494 & 0,309 & 0,659 & 0,281 & $0,0 \%$ & $33,3 \%$ & $66,7 \%$ \\
\hline Magdalena del Mar & 0,500 & 0,526 & 0,284 & 0,206 & 0,750 & $15,0 \%$ & $65,0 \%$ & $20,0 \%$ \\
\hline Miraflores & 0,745 & 0,756 & 0,513 & 0,201 & 0,902 & $52,0 \%$ & $28,0 \%$ & $20,0 \%$ \\
\hline Pachacámac & 0,442 & 0,653 & 0,390 & 0,739 & 0,486 & $0,0 \%$ & $47,8 \%$ & $52,2 \%$ \\
\hline Pucusana & 0,335 & 0,502 & 0,253 & 0,753 & 0,230 & $0,0 \%$ & $42,9 \%$ & $57,1 \%$ \\
\hline Pueblo Libre & 0,556 & 0,629 & 0,355 & 0,153 & 0,802 & $40,0 \%$ & $45,0 \%$ & $15,0 \%$ \\
\hline Puente Piedra & 0,395 & 0,470 & 0,207 & 0,632 & 0,242 & $0,0 \%$ & $42,3 \%$ & $57,7 \%$ \\
\hline Punta Hermosa & 0,000 & 0,000 & 0,000 & 0,686 & 0,000 & $0,0 \%$ & $10,0 \%$ & $0,0 \%$ \\
\hline Punta Negra & 0,498 & 0,550 & 0,302 & 0,728 & 0,206 & $0,0 \%$ & $50,0 \%$ & $50,0 \%$ \\
\hline Rímac & 0,444 & 0,517 & 0,335 & 0,425 & 0,531 & $0,0 \%$ & $58,9 \%$ & $41,1 \%$ \\
\hline San Bartolo & 0,188 & 0,135 & 0,156 & 0,591 & 0,097 & $0,0 \%$ & $33,3 \%$ & $66,7 \%$ \\
\hline San Borja & 0,674 & 0,702 & 0,385 & 0,202 & 0,862 & $47,4 \%$ & $36,8 \%$ & $15,8 \%$ \\
\hline San Isidro & 0,737 & 0,747 & 0,487 & 0,197 & 0,929 & $66,7 \%$ & $26,7 \%$ & $6,7 \%$ \\
\hline San Juan de Lurigancho & 0,415 & 0,499 & 0,294 & 0,554 & 0,402 & $0,3 \%$ & $47,1 \%$ & $52,6 \%$ \\
\hline San Juan de Miraflores & 0,415 & 0,466 & 0,302 & 0,493 & 0,453 & $0,7 \%$ & $58,1 \%$ & $41,2 \%$ \\
\hline San Luis & 0,428 & 0,451 & 0,289 & 0,430 & 0,464 & $5,6 \%$ & $38,9 \%$ & $55,6 \%$ \\
\hline San Martín de Porres & 0,440 & 0,439 & 0,234 & 0,376 & 0,480 & $0,8 \%$ & $68,3 \%$ & $30,9 \%$ \\
\hline San Miguel & 0,689 & 0,525 & 0,459 & 0.263 & 0,837 & $23,3 \%$ & $65,1 \%$ & $11,6 \%$ \\
\hline Santa Anita & 0,399 & 0,473 & 0,244 & 0,424 & 0,416 & $0,0 \%$ & $67,1 \%$ & $32,9 \%$ \\
\hline Santa María del Mar & 0,000 & 0,000 & 0,000 & 0,000 & 0,400 & $0,0 \%$ & $0,0 \%$ & $0,0 \%$ \\
\hline Santa Rosa & 0,627 & 0,734 & 0,508 & 0,824 & 0,307 & $20,0 \%$ & $50,0 \%$ & $30,0 \%$ \\
\hline Santiago de Surco & 0,755 & 0,720 & 0,556 & 0,326 & 0,874 & $48,1 \%$ & $37,7 \%$ & $14,3 \%$ \\
\hline Surquillo & 0,493 & 0,639 & 0,352 & 0,256 & 0,746 & $16,7 \%$ & $66,7 \%$ & $16,7 \%$ \\
\hline Villa el Salvador & 0,351 & 0,437 & 0,242 & 0,495 & 0,334 & $0,0 \%$ & $55,4 \%$ & $44,6 \%$ \\
\hline Villa Maria del Triunfo & 0,410 & 0,481 & 0,292 & 0,580 & 0,329 & $0,0 \%$ & $52,0 \%$ & $48,0 \%$ \\
\hline Bellavista & 0,545 & 0,473 & 0,264 & 0,216 & 0,692 & $8,1 \%$ & $81,1 \%$ & $10,8 \%$ \\
\hline Callao & 0,376 & 0,445 & 0,256 & 0,388 & 0,449 & $0,7 \%$ & $64,2 \%$ & $35,1 \%$ \\
\hline Carmen de la Legua & 0,388 & 0,398 & 0,249 & 0,376 & 0,423 & $0,0 \%$ & $56,2 \%$ & $43.8 \%$ \\
\hline La Perla & 0,602 & 0,593 & 0,403 & 0,358 & 0,702 & $0,0 \%$ & $76,5 \%$ & $23,5 \%$ \\
\hline La Punta & 0,525 & 0,601 & 0,412 & 0,387 & 0,630 & $0,0 \%$ & $50,0 \%$ & $50,0 \%$ \\
\hline Mi Perú & 0,284 & 0,426 & 0,166 & 0,639 & 0,150 & $0,0 \%$ & $46,7 \%$ & $53,3 \%$ \\
\hline Ventanilla & 0,432 & 0,621 & 0,361 & 0,727 & 0,349 & $0,0 \%$ & $47,0 \%$ & $53,0 \%$ \\
\hline
\end{tabular}


Tabla B2

Indicadores de segregación en $2{ }^{\circ}$ grado de secundaria, según distrito

\begin{tabular}{|c|c|c|c|c|c|c|c|c|}
\hline \multirow{2}{*}{ Distrito } & \multicolumn{2}{|c|}{ Disimilitud } & \multirow{2}{*}{ ICC } & \multicolumn{2}{|c|}{ Aislamiento } & \multicolumn{3}{|c|}{ Grado de segregación } \\
\hline & $\mathrm{MB}+\mathrm{B}$ & Alto & & $\mathrm{MB}+\mathrm{B}$ & Alto & Muy hom & Hom & Het \\
\hline Ancón & 0,312 & 0,439 & 0,323 & 0,695 & 0,162 & $0,0 \%$ & $50,0 \%$ & $50,0 \%$ \\
\hline Ate & 0,392 & 0,485 & 0,270 & 0,527 & 0,432 & $1,1 \%$ & $43,7 \%$ & $55,2 \%$ \\
\hline Barranco & 0,358 & 0,445 & 0,244 & 0,297 & 0,532 & $0,0 \%$ & $65,0 \%$ & $35,0 \%$ \\
\hline Breña & 0,420 & 0,380 & 0,175 & 0,232 & 0,573 & $0,0 \%$ & $70,6 \%$ & $29,4 \%$ \\
\hline Carabayllo & 0,446 & 0,435 & 0,282 & 0,589 & 0,335 & $0,0 \%$ & $48,9 \%$ & $51,1 \%$ \\
\hline Chaclacayo & 0,317 & 0,434 & 0,200 & 0,405 & 0,396 & $0,0 \%$ & $68,4 \%$ & $31,6 \%$ \\
\hline Chorrillos & 0,486 & 0,506 & 0,321 & 0,414 & 0,567 & $2,4 \%$ & $52,9 \%$ & $44,7 \%$ \\
\hline Cieneguilla & 0,318 & 0,329 & 0,182 & 0,560 & 0,252 & $0,0 \%$ & $44,4 \%$ & $55,6 \%$ \\
\hline Comas & 0,352 & 0,362 & 0,229 & 0,401 & 0,389 & $0,7 \%$ & $53,4 \%$ & $45,9 \%$ \\
\hline El Agustino & 0,342 & 0,335 & 0,171 & 0,465 & 0,281 & $0,0 \%$ & $57,5 \%$ & $42,5 \%$ \\
\hline Independencia & 0,327 & 0,334 & 0,196 & 0,464 & 0,265 & $0,0 \%$ & $47,9 \%$ & $52,1 \%$ \\
\hline Jesús María & 0,449 & 0,398 & 0,158 & 0,204 & 0,621 & $0,0 \%$ & $82,6 \%$ & $17,4 \%$ \\
\hline La Molina & 0,657 & 0,596 & 0,354 & 0,432 & 0,781 & $26,4 \%$ & $56,6 \%$ & $17,0 \%$ \\
\hline La Victoria & 0,397 & 0,440 & 0,230 & 0,437 & 0,439 & $0,0 \%$ & $61,9 \%$ & $38,1 \%$ \\
\hline Lima & 0,368 & 0,393 & 0,230 & 0,337 & 0,481 & $1,2 \%$ & $51,2 \%$ & $47,6 \%$ \\
\hline Lince & 0,438 & 0,322 & 0,166 & 0,202 & 0,569 & $0,0 \%$ & $73,7 \%$ & $26,3 \%$ \\
\hline Los Olivos & 0,454 & 0,419 & 0,217 & 0,365 & 0,499 & $0,0 \%$ & $63,0 \%$ & $37,0 \%$ \\
\hline Lurigancho & 0,487 & 0,491 & 0,343 & 0,654 & 0,353 & $0,0 \%$ & $37,0 \%$ & $63,0 \%$ \\
\hline Lurín & 0,339 & 0,426 & 0,165 & 0,603 & 0,257 & $0,0 \%$ & $26,1 \%$ & $73,9 \%$ \\
\hline Magdalena del Mar & 0,493 & 0,433 & 0,198 & 0,272 & 0,596 & $0,0 \%$ & $80,0 \%$ & $20,0 \%$ \\
\hline Miraflores & 0,538 & 0,611 & 0,296 & 0,258 & 0,754 & $16,7 \%$ & $66,7 \%$ & $16,7 \%$ \\
\hline Pachacámac & 0,368 & 0,429 & 0,334 & 0,710 & 0,252 & $0,0 \%$ & $37,8 \%$ & $62,2 \%$ \\
\hline Pucusana & 0,380 & 0,257 & 0,242 & 0,766 & 0,064 & $0,0 \%$ & $16,7 \%$ & $83,3 \%$ \\
\hline Pueblo Libre & 0,541 & 0,467 & 0,237 & 0,168 & 0,755 & $11,1 \%$ & $61,1 \%$ & $27,8 \%$ \\
\hline Puente Piedra & 0,347 & 0,364 & 0,164 & 0,598 & 0,200 & $0,0 \%$ & $42,0 \%$ & $58,0 \%$ \\
\hline Punta Hermosa & 0,000 & 0,000 & 0,000 & 0,714 & 0,057 & $0,0 \%$ & $0,0 \%$ & $10,0 \%$ \\
\hline Punta Negra & 0,383 & 0,360 & 0,183 & 0,643 & 0,191 & $0,0 \%$ & $25,0 \%$ & $75,0 \%$ \\
\hline Rímac & 0,348 & 0,364 & 0,233 & 0,409 & 0,382 & $0,0 \%$ & $46,5 \%$ & $53,5 \%$ \\
\hline San Bartolo & 0,000 & 0,000 & 0,000 & 0,538 & 0,175 & $0,0 \%$ & $0,0 \%$ & $10,0 \%$ \\
\hline San Borja & 0,631 & 0,555 & 0,229 & 0,241 & 0,787 & $16,7 \%$ & $77,8 \%$ & $5,6 \%$ \\
\hline San Isidro & 0,649 & 0,519 & 0,199 & 0,164 & 0,812 & $12,5 \%$ & $81,2 \%$ & $6,2 \%$ \\
\hline San Juan de Lurigancho & 0,378 & 0,412 & 0,219 & 0,505 & 0,344 & $0,0 \%$ & $42,6 \%$ & $57,4 \%$ \\
\hline San Juan de Miraflores & 0,346 & 0,393 & 0,240 & 0,439 & 0,390 & $1,1 \%$ & $45,3 \%$ & $53,7 \%$ \\
\hline San Luis & 0,382 & 0,438 & 0,206 & 0,385 & 0,447 & $0,0 \%$ & $64,7 \%$ & $35,3 \%$ \\
\hline San Martín de Porres & 0,395 & 0,358 & 0,176 & 0,348 & 0,433 & $0,0 \%$ & $63,2 \%$ & $36,8 \%$ \\
\hline San Miguel & 0,429 & 0,382 & 0,251 & 0,214 & 0,684 & $3,9 \%$ & $86,3 \%$ & $9,8 \%$ \\
\hline Santa Anita & 0,362 & 0,370 & 0,189 & 0,381 & 0,376 & $0,0 \%$ & $60,0 \%$ & $40,0 \%$ \\
\hline Santa Rosa & 0,709 & 0,710 & 0,604 & 0,817 & 0,403 & $0,0 \%$ & $71,4 \%$ & $28,6 \%$ \\
\hline Santiago de Surco & 0,613 & 0,527 & 0,296 & 0,286 & 0,765 & $15,9 \%$ & $68,1 \%$ & $15,9 \%$ \\
\hline Surquillo & 0,345 & 0,409 & 0,179 & 0,275 & 0,532 & $0,0 \%$ & $69,2 \%$ & $30,8 \%$ \\
\hline Villa el Salvador & 0,304 & 0,331 & 0,165 & 0,436 & 0,274 & $0,0 \%$ & $55,9 \%$ & $44,1 \%$ \\
\hline Villa María del Triunfo & 0,333 & 0,378 & 0,209 & 0,505 & 0,255 & $0,0 \%$ & $44,0 \%$ & $56,0 \%$ \\
\hline Bellavista & 0,373 & 0,402 & 0,176 & 0,260 & 0,528 & $0,0 \%$ & $62,5 \%$ & $37,5 \%$ \\
\hline Callao & 0,352 & 0,384 & 0,193 & 0,379 & 0,396 & $0,0 \%$ & $57,7 \%$ & $42,3 \%$ \\
\hline Carmen de la Legua & 0,188 & 0,187 & 0,076 & 0,261 & 0,293 & $0,0 \%$ & $85,7 \%$ & $14,3 \%$ \\
\hline La Perla & 0,461 & 0,315 & 0,167 & 0,281 & 0,584 & $0,0 \%$ & $66,7 \%$ & $33,3 \%$ \\
\hline La Punta & 0,086 & 0,350 & 0,053 & 0,066 & 0,577 & $0,0 \%$ & $10,0 \%$ & $0,0 \%$ \\
\hline Mi Perú & 0,292 & 0,323 & 0,139 & 0,625 & 0,118 & $0,0 \%$ & $40,0 \%$ & $60,0 \%$ \\
\hline Ventanilla & 0,456 & 0,570 & 0,312 & 0,730 & 0,305 & $0,0 \%$ & $36,9 \%$ & $63,1 \%$ \\
\hline
\end{tabular}

